This item was submitted to Loughborough's Research Repository by the author.

Items in Figshare are protected by copyright, with all rights reserved, unless otherwise indicated.

\title{
Insertion repair
}

PLEASE CITE THE PUBLISHED VERSION

http://dx.doi.org/10.1080/08351813.2011.544136

PUBLISHER

(c) Taylor \& Francis Group

VERSION

AM (Accepted Manuscript)

LICENCE

CC BY-NC-ND 4.0

REPOSITORY RECORD

Wilkinson, Susan J., and Ann Weatherall. 2019. "Insertion Repair”. figshare. https://hdl.handle.net/2134/15594. 
This item was submitted to Loughborough's Institutional Repository (https://dspace.lboro.ac.uk/) by the author and is made available under the following Creative Commons Licence conditions.

\section{creative
commons}

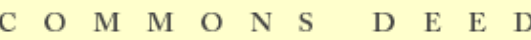

Attribution-NonCommercial-NoDerivs 2.5

You are free:

- to copy, distribute, display, and perform the work

Under the following conditions:

Attribution. You must attribute the work in the manner specified b the author or licensor.

Noncommercial. You may not use this work for commercial purposes.

No Derivative Works. You may not alter, transform, or build upon this work.

- For any reuse or distribution, you must make clear to others the license terms of this work.

- Any of these conditions can be waived if you get permission from the copyright holder.

Your fair use and other rights are in no way affected by the above.

This is a human-readable summary of the Leqal Code (the full license).

\section{Disclaimer 만}

For the full text of this licence, please go to: http://creativecommons.org/licenses/by-nc-nd/2.5/ 
Wilkinson, S. and Weatherall, A. (2011) Insertion Repair. Research on Language and Social Interaction, 44(1): 65-91

\title{
INSERTION REPAIR
}

\author{
Sue Wilkinson, Loughborough University
}

and

Ann Weatherall, Victoria University of Wellington

Short title: Insertion repair

Address for correspondence:

Sue Wilkinson

Department of Social Sciences

Loughborough University

Loughborough

Leicestershire LE11 3TU, UK

<s.wilkinson@lboro.ac.uk>

Tel. $+44(0) 1509223365$

Fax. +44 (0)1509 223944

Acknowledgements:

This work was partially supported by a grant to both authors from the (NZ) ISAT Bilateral Research Activities programme. An early version was presented by Sue Wilkinson as a keynote address at the 2009 Language and Social Interaction (LSI) Conference at the University of California, Santa Barbara, USA and she would like to thank the Conference Committee and the Department of Sociology at UCSB for their financial support. Thanks are also due to the LSI conference audience and to Gene Lerner, Sandra Thompson and Emanuel Schegloff for their very helpful feedback. The authors would particularly like to thank Celia Kitzinger for sharing data from the Birth Crisis, Home Birth and Pelvic Partnership data sets, and for her valuable comments on many of the analyses included here. Any remaining errors are, of course, the responsibility of the authors. 


\title{
INSERTION REPAIR
}

\begin{abstract}
Insertion repair is a practice in which a speaker halts their talk-in-progress to go back and add something else into the turn before resuming (e.g. inserting blind in this girl's fixed up on a da- a blind date). This article provides the first systematic overview of insertion repair, based on an analysis of more than 500 instances. We first describe the technology of insertion repair; then identify its the main actions. The most common action is specifying: modifying an original reference formulation so as to identify a particular type of referent, or a unique referent, in order to distinguish between possible referents, or in the service of the interactional task-at-hand. A second common action is intensifying: modifying the original formulation so as strengthen it. Less common actions are describing, correcting, adjusting, and expanding. Finally we consider the relevance of our findings for conversation analytic work on repair, referring, and the relationship between grammar and action.
\end{abstract}

Keywords: conversation analysis, formulation, referring, repair, specifying, talk-ininteraction, intensifying, inserting 


\section{INSERTION REPAIR}

There is a range of practices for dealing with problems in speaking, hearing or understanding talk-in-interaction, many of which have been described by conversation analysts under the general rubric of repair (e.g. Schegloff, Jefferson and Sacks, 1977; Schegloff, 2000a). Insertion repair was initially identified some thirty years ago by Schegloff (1979), and is one of a number of different repair operations (others of which include replacing, deleting, searching, reformatting, and reordering [Schegloff, 2008]). Extracts 1 and 2 show instances of insertion.

(1) this girl's fixed up on a da- a blind dạ:te. ${ }^{i}$ (\#216)

(2) he's made a calc- .h an electoral calculation (\#725)

In each of these extracts a speaker stops their talk at a point where it cannot be possibly complete (in the middle of a word) in order to go back and add something else into the talk, before resuming the utterance that was originally in progress. In (1), the speaker stops the talk part way through the word that turns out to be date, in order to insert the word blind; in (2), the speaker stops the talk part way through the word that turns out to be calculation, in order to insert the word electoral. (Inserted material is shown in bold typeface throughout.) The repaired formulations are a blind date (1) and an electoral calculation (2).

As Schegloff has said recently: 
inserting is done by a speaker who cannot wait, or will not wait, for the end of what they are in the middle of saying to get something else said or included as part of it. Rather they override the preference for progressivity [...] to stop the talk to insert something else into it. What sorts of things, we may ask, warrant such an override, warrant such a marked usage? (Schegloff, 2008)

This article addresses - and extends - Schegloff's question. It is based on a systematic analysis of a collection of more than five hundred ${ }^{\mathrm{ii}}$ insertion repairs, compiled from a wide range of British, New Zealand and US English data sets. ${ }^{\text {iii }}$ We first sketch out the technology of insertion repair, with special reference to what gets inserted. We then identify the key actions that the practice of insertion repair is used to accomplish.

\section{The Technology of Insertion Repair}

Insertion repair is overwhelmingly initiated in the same turn-constructional unit (TCU) as the the word that is modified by the inserted material. This is typical for repair more generally: "self-initiated same turn repair is, by far, the most common form of repair" (Schegloff 1979: 268). Around three-quarters of the instances in our collection are same-TCU repairs - and in most of these, not only the repair initiation but also the repair solution is within the same TCU. We will focus here on insertion repairs begun - and completed - within a single TCU, since these account for the vast majority of instances ${ }^{\text {iv }}$.

The insertion repair segment begins at the point at which a speaker signals to a recipient that what follows may not be more of what had immediately preceded (the repair initiation). In 
Extracts 1 and 2, the repair initiation is a cut-off (on the words date and calculation) and what follows is a discontinuous element (i.e. something other than a continuation of the word that has been started). In both cases, this is a repeat of the indefinite article $a$ (modified to $a n$ in [2]) - which constitutes the pre-frame. The pre-frame comes before the new material (blind and electoral). The repair segment ends when the talk previously in progress is resumed, as evidenced by the repeat or return of some earlier part of the talk which serves to post-frame the inserted material: the sound $d a$ (the beginning of date) in (1); the sound calc (the beginning of calculation) in (2). It is this repeated or returning element - which post-frames the inserted material - that makes of a repair an insertion rather than some other kind of repair operation (Schegloff, 2008). The repair solution in Extracts 1 and 2 is the whole of the modified formulation, (composed of pre-frame, inserted material, and post-frame): a blind date, an electoral calculation.

We will sketch out the technology of insertion repair by making some observations - across our data collection - about the repair initiation, the pre- and post-frames, the inserted material, and the repair solution in insertion repair.

\section{Repair initiation}

Speakers usually initiate (what turns out to be) insertion repair either in - or just after - the word before which the new material is to be inserted. In Extracts 1 and 2 the repair initiation (in both cases a cut-off) is on the word before which the new material is to be inserted: $d a$ (in 1), calc- (in 2). In Extracts 3 and 4 speakers initiate repair just after the word before which the new material is to be inserted: on (as is typical in such cases) the very next word following it. Only very occasionally is repair initiated later than this.

(3) we walked th- we only walked the 


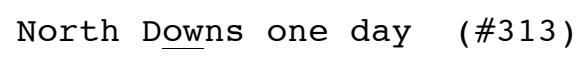

A cut-off (linguistically a 'stop' - glottal, dental, alveolar, etc.) is the most common kind of repair initiation, used in around three-quarters of the cases in our collection. (It is also very common in self-initiated repair more generally: its use does not indicate the type of repair operation being initiated, nor indeed is it necessarily followed by repair, Schegloff and Lerner, 2009.) Speakers can initiate (what turns out to be) insertion repair with a cut-off at any point in the production of a word: very early (as in Extract 10 below); part-way through (as in Extracts 1 and 2) or right at the end of its production (as in Extract 4). It can also be initiated with sound stretches (e.g. the elongation of factory in Extract 5; see also 12 and 28); pauses (e.g. after bring in Extract 6); and others of those features of talk identified by conversation analysts (e.g. Schegloff, 2008) as possible ways of initiating self-repair.

(5) [At the] moment my factory: manufacturing factory here is sort'v at a stạ:ndstill:: (\#260)

(6) It may bring (.) may well bring the temperature dow $: \mathrm{n} \cdot \quad(\# 476)$

It is also quite common for insertion repair to be launched without any explicit repair initiation: that is, there is no alert to the recipient that the next thing the speaker says might not be continuous with what has been said up to that point. Instead, the recipient of that 'next thing' finds it analyzably discontinuous and hence comes to understand that repair may be underway (Lerner and Kitzinger, 2010). In common with cases where repair is explicitly 
initiated, it is not apparent to the recipient that an insertion repair, in particular, has been effected until the repeat or return of an earlier element of the utterance. Extracts 7 and 8 show insertion repairs launched without explicit initiation: inserting complaints before procedure (7) and Islamic before chap (8) ${ }^{\mathrm{v}}$.

(7) you know the procedure complaints procedure goes out the window thuh minute you take legal action. (\#599)

(8) I have this $u: h$ this chap this: Islamic chap who:'s into: Yunani medicine (\#526)

\section{Pre- and post-frames}

In 6 of the 8 instances displayed so far, the repair solution is both pre- and post-framed: that is, some part of the previous talk is repeated before the inserted material, and some other part of the previous talk is repeated after it. So in Extract 6 (may bring (.) may well bring), the inserted material well is pre-framed by may and post-framed by bring; and in Extract 8 (this chap this Islamic chap) the inserted material Islamic is pre-framed by this and post-framed by chap; see also 1-4. In pre-framing an insertion repair speakers typically repeat just one word from the prior talk. ${ }^{\mathrm{vi}}$

However, insertion repairs are very often not pre-framed. In Extract 5 there is no repeat of $m y$ to pre-frame manufacturing (my factory: manufacturing factory); and in Extract 7 there is no repeat of the definite article the to pre-frame complaints (the procedure complaints procedure). Likewise, in Extracts 9-12 - and indeed, in well over half of the instances in our collection - the inserted material is post-framed only.

(9) I've really gotta get this: t- roo:m til:died. (\#213) 


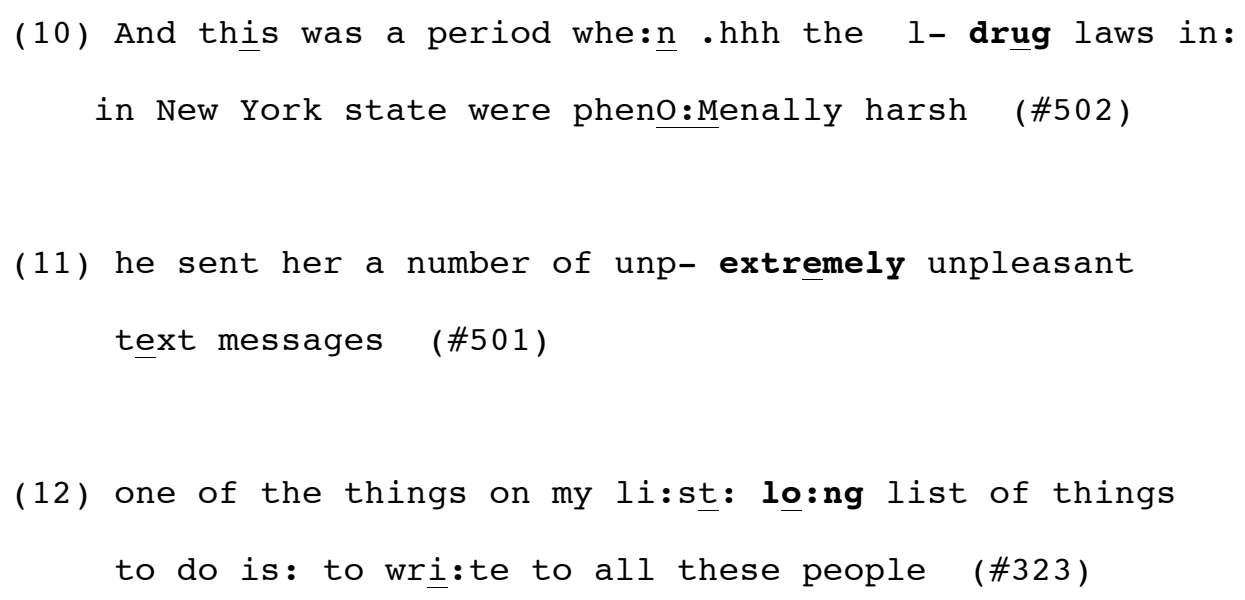

Since a post-frame is constituted by the return of some element of the talk produced prior to repair initiation, its size is directly related to where repair was initiated. Post-frames range from a tiny sound (as in Extract 9 [the first part of the first sound of the word tidied, which post-frames the inserted word room]) to a whole word or more ${ }^{\text {vii }}$ (as in Extract 12 [list, which post-frames the inserted word long]).

\section{Inserted material}

It is overwhelmingly the case that the inserted material is a single word (as with blind [1], electoral [2], only [3], physical [4], and so on for all the data shown so far). However, speakers can insert more than a single word, or less than a single word. Insertions of more than a single word tend to be idiomatic (Extract 13) or otherwise effectively single - albeit multi-word - units (Extracts 14 and 15) viii $^{\text {vii }}$

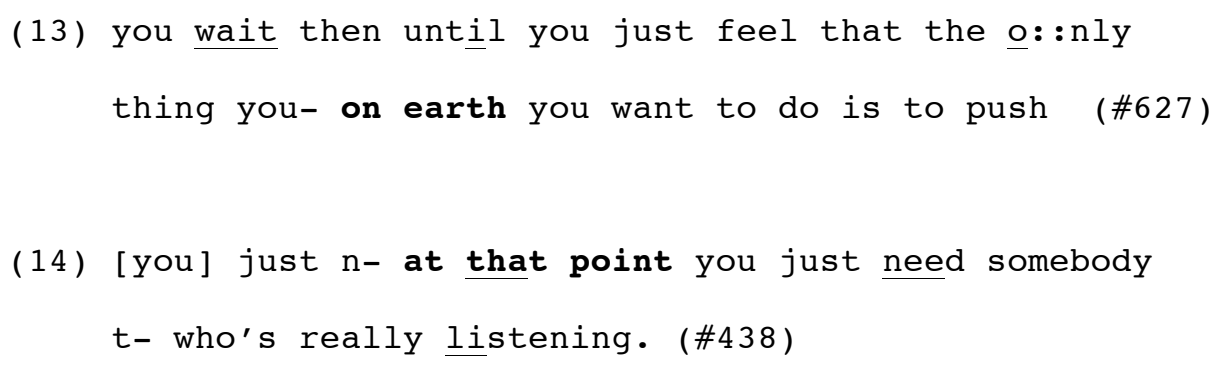




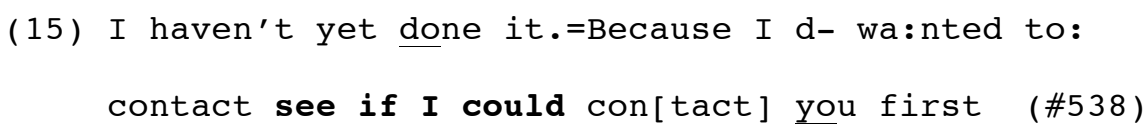

Insertions of less than a word commonly include prefixes and initial components of complex words: ex- before consultant (16); fore before head (17); neuro before chemicals (18); ear before ring (19); play before school (20).

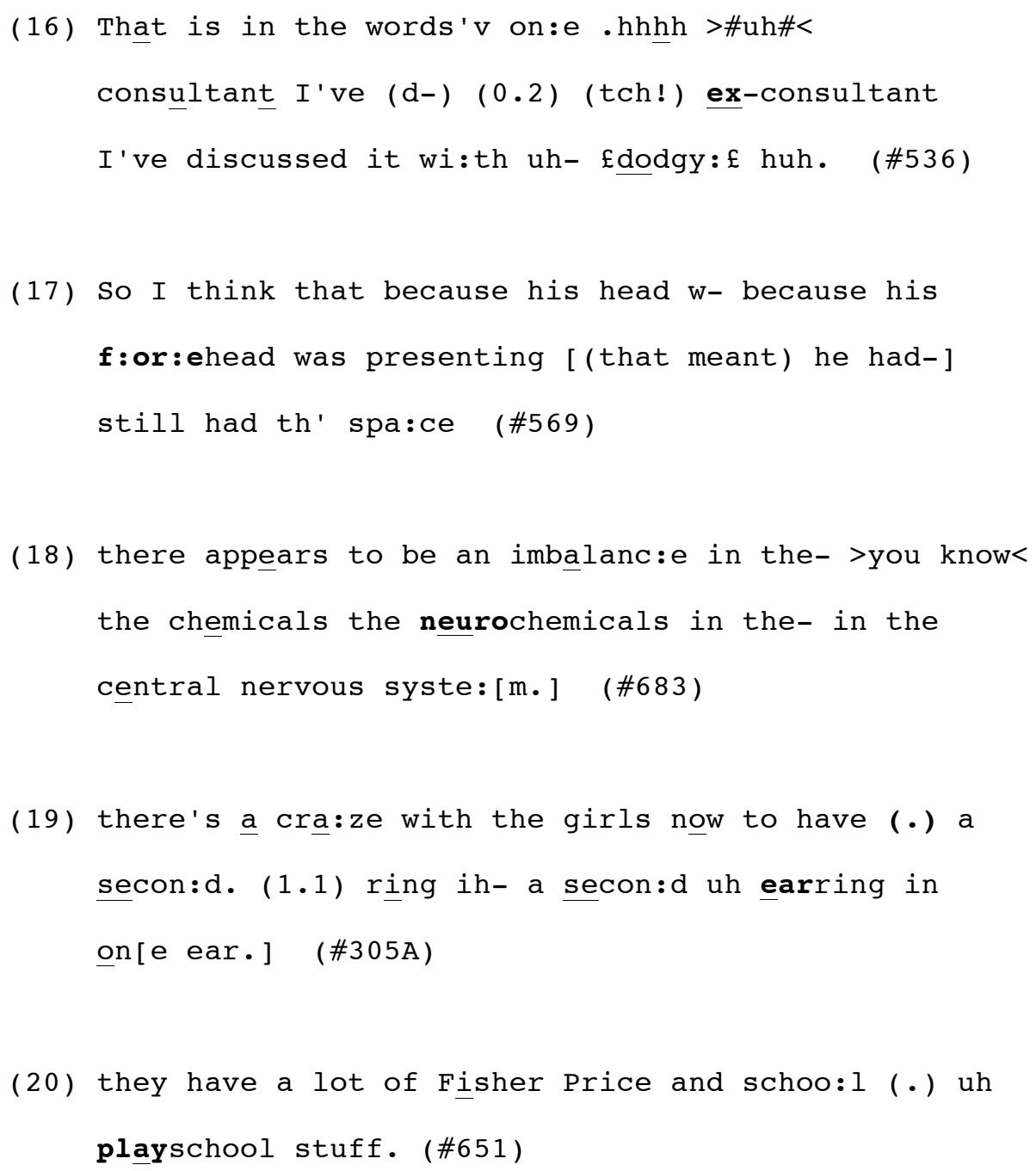

As far as we know, speakers can insert material in any grammatical form. This includes (in order of their frequency in our collection):

- adjectives (e.g. electoral [2], physical [4], Islamic [8], long [12]) - these 
constitute nearly half of the inserted material in our collection;

- adverbs (e.g. only [3], extremely [11]) - constituting more than a quarter;

- prefixes and other initial morphemes (e.g. ex- [16], neuro [18]);

- prepositionals and prepositional phrases (e.g. at that point [14]);

- verb and verb phrases (e.g. see if I could [15]);

- nouns and pronouns (e.g. room [9]);

- other grammatical forms (e.g. initializations [22] and interjections).

\section{Repair solution}

One important and distinctive feature of insertion repair compared with other repair operations is that a formulation is modified, rather than deleted or replaced. So, the repair solution a blind date (1) retains the original formulation date; my long list (12) retains list; and playschool (20) retains school. In retaining the original formulation as part of the repair solution, the speaker treats it as not wholly wrong (and so not in need of deleting or replacing), but as in need of modification. In each case the repair solution provides a modified version of the original formulation, rather than removing it altogether (as in deletion repair) or substituting some other formulation for it (as in replacement repair). As we will see in the next section, this technical feature of insertion repair is profoundly consequential for the kinds of actions speakers are able to use it to accomplish.

\section{The Actions of Insertion Repair}

Insertion repair is used to modify (rather than to delete or to replace) something that the speaker has already said or started to say. The most common way in which the inserted 
material modifies the original formulation is by specifying it more closely. Specifying is the repairing action that accounts for well over half of all the instances in our collection. A second repairing action is intensifying (around a quarter of our collection). There is also a range of other (less common) repairing actions - describing, correcting, adjusting and expanding the original formulation - which are discussed in the last section.

\section{(1) Specifying:}

In what we are calling specifying, the inserted material specifies a referent more closely, modifying an original reference formulation so as to identify a unique referent or a particular type of referent.

Reference formulations modified in this way include references to places (e.g. the cemetery [21]); persons (e.g. this chap [26]); and objects (e.g. the tube [27]). The inserted material serves to define or identify the referent more specifically, or to restrict the scope of the original reference formulation by more closely specifying which one or which type of referent is intended. In this way, the repaired reference formulation specifies the referent.

Some insertion repairs identify a unique referent - as for example when the referent of the cemetery is specified as one particular cemetery by inserting the proper name Cary. Other insertion repairs identify the referent as one of a type - as for example when the referent of this chap is specified as a particular type of chap by inserting the defining adjective Islamic; and the referent of the tube is specified as a particular type of tube by inserting the defining adjective feeding. ${ }^{\mathrm{ix}}$ 
As we have noted earlier, one distinctive feature of insertion repair is that the original formulation is retained as part of the repair solution. Another way of saying this is that it does not simply follow the repaired reference formulation as a post-frame, but is an integral part of it. So, the modified reference formulation the Cary cemetery (21) retains the original reference cemetery; this Islamic chap (26) retains chap; and the feeding tube (27) retains tube. In repairing the original formulation in this way, the speaker treats it as not wholly wrong (and so not in need of deleting or replacing), but as insufficiently specific. In each case the repair solution increases the specificity of reference.

When a speaker disrupts the smooth progressivity of their talk to increase specificity of reference, the issue - for both recipient and analyst - is how this further specification is relevant:

A fundamental property of all versions, descriptions, accounts and claims about events, is that speakers construct them to be as precise or exact as they need to be - as is relevant - for the interactional contexts in which they are produced, and for the interactional tasks at hand. (Drew, 2003: 936)

In using insertion repair to specify a referent, a speaker treats their original reference formulation as insufficiently precise or exact: the repair is designed to specify the referent more precisely or exactly.

Of course, virtually any mention (of a person, place, or object) can be treated by participants (or analyst) as insufficiently precise or exact. But in some cases this lack of precision is not an abstract or principled possibility: rather the imprecision is occasioned by some prior mention in the preceding talk of a referent with whom (or with which) the current referent might plausibly be confused: in the data to be shown, another cemetery, another antenatal 
teacher, or another kind of wash. In other words, what is centrally implicated in such instances is not that there is some/any other possible referent in the world, but that there is a particular, locally-active alternative to the one to which the speaker means to refer. This renders the original formulation insufficiently precise to differentiate between two (or more) possible referents and the relevance of the insertion is to address a possible problem in understanding, pre-empting a potential difficulty in identifying the correct referent. ${ }^{\mathrm{x}}$ This use of insertion repair is exemplified in Extracts 21-25 below.

However, there are also many instances (exemplified in Extracts 26-31 below) in which insertion repairs doing specifying are not warranted by possible understanding problems occasioned by locally-active referents in the immediately prior talk. In such instances it is solely the interactional task-at-hand which underwrites a specification of the referent. This latter - task-oriented - use of specifying is also repair, but here it is used in the service of the action of the turn-in-progress (whatever that action may be).

\section{(a) Specifying to differentiate between possible (locally-occurring) alternative referents}

In each of the instances below, there is a locally-occurring source of possible misunderstanding: that is, an alternative possible referent to the one to which the speaker means to refer has been mentioned, or is analyzably inferable. The inserted material is designed to enable a recipient to understand to which of these possible referents a speaker is referring. In each of the following extracts, the referent is a particular place, person, object or type of object, and in each, the speaker displays an orientation to the possibility that the reference formulation originally selected may not serve adequately to identify the intended 
referent, but may be taken instead to refer to a different place, person, object or type of object. The repair is analyzably designed to differentiate between these possible referents.

In Extract 21, the speaker Philip - whose elderly mother has just died - is telling his friend (Lesley) about the arrangements for the funeral and burial. The insertion repair is at lines 1112, where Philip says of the burial (though the words 'burial' and 'buried' are 'silent improprieties', never actually articulated [c.f. Schegloff, 2003: 251]) that it'll be in the cehthe Cary cemetery. Here, the proper name $\mathrm{Cary}^{x i}$ is inserted to modify the original reference formulation, specifying the referent as the Cary cemetery.

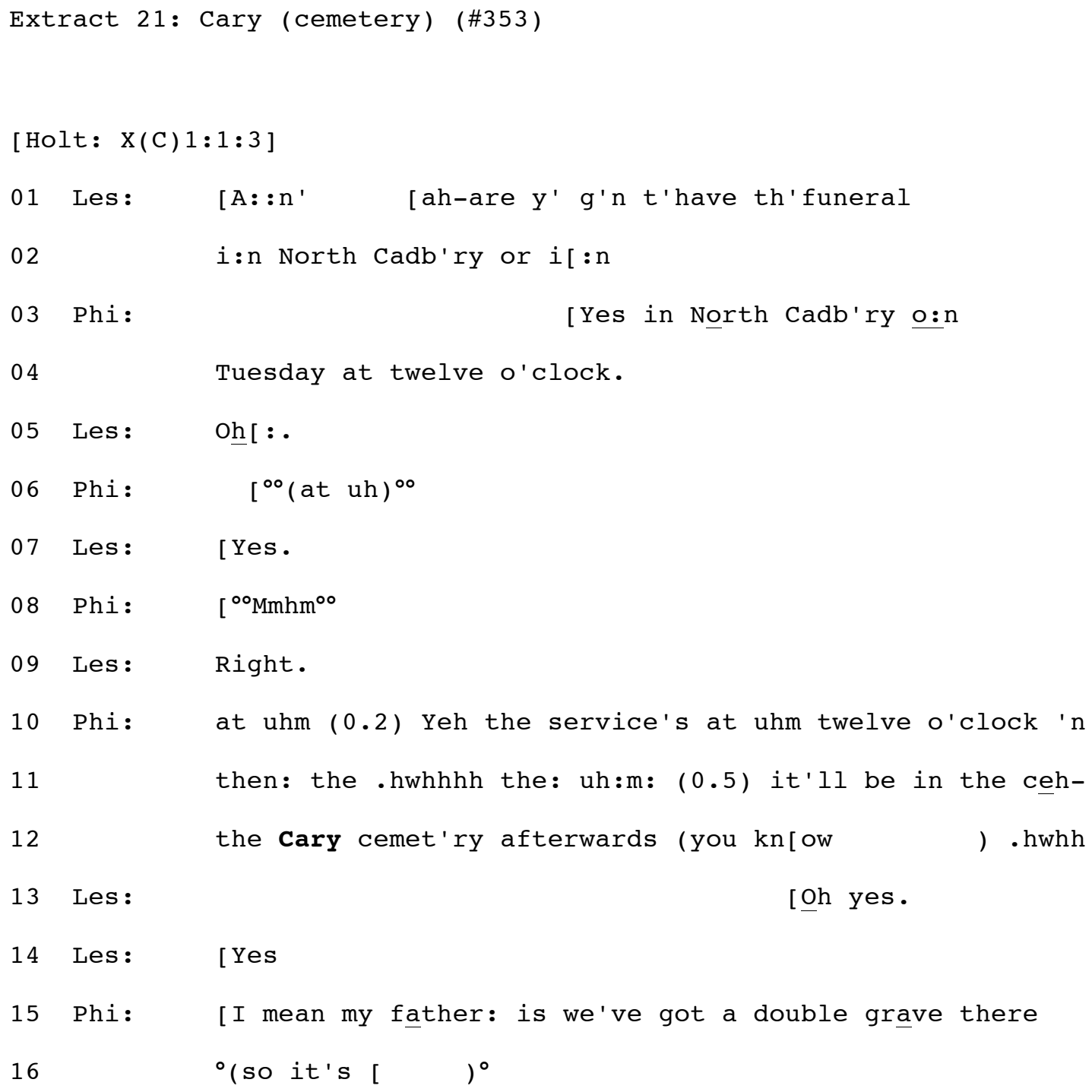


The locally-active alternative referent to Cary cemetery is North Cadbury cemetery (possibly inferable from the location of the funeral). In the course of informing Lesley about the location of the burial, Philip hears that the reference formulation he first selected, the cemetery (line 11) is vulnerable to being heard as referring to a different cemetery (the North Cadbury cemetery), since he has just (at line 3) confirmed North Cadbury as the location for the funeral service. He cuts off the cemetery in the course of its production (the ceh-, line 11) to insert the name Cary, identifying the referent more specifically as the Cary cemetery (line 12) (and retaining the original reference formulation cemetery as part of the repair solution). The specification of the referent as the Cary cemetery (in particular) differentiates between two possible referents. Through identifying a unique referent, the speaker pre-empts a possible misunderstanding.

In Extract 22, from a call to a helpline for women in crisis after a traumatic birth, the caller is answering a question from the call-taker about her midwife - a question asked in the service of finding out who had advised her to call the crisis line. The insertion repair is at lines $15-16$ where the caller, Jennifer, says It was actually my an- my NCT antenatal teacher. Here NCT (an initialization of National Childbirth Trust ${ }^{\mathrm{xii}}$ ) is inserted to modify the compound reference formulation antenatal teacher, specifying the referent as my NCT antenatal teacher.

Extract 22: NCT (antenatal teacher) (\#408)

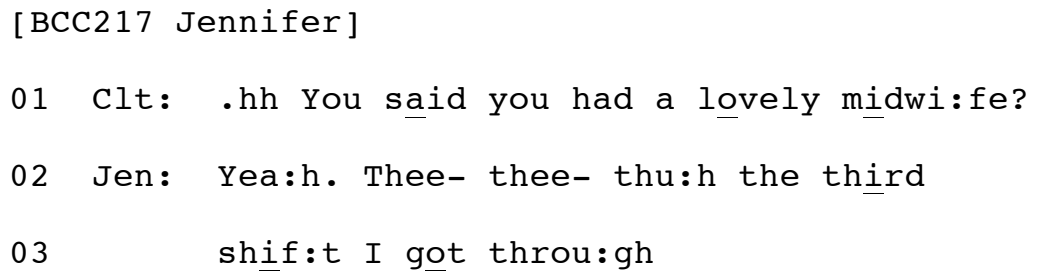




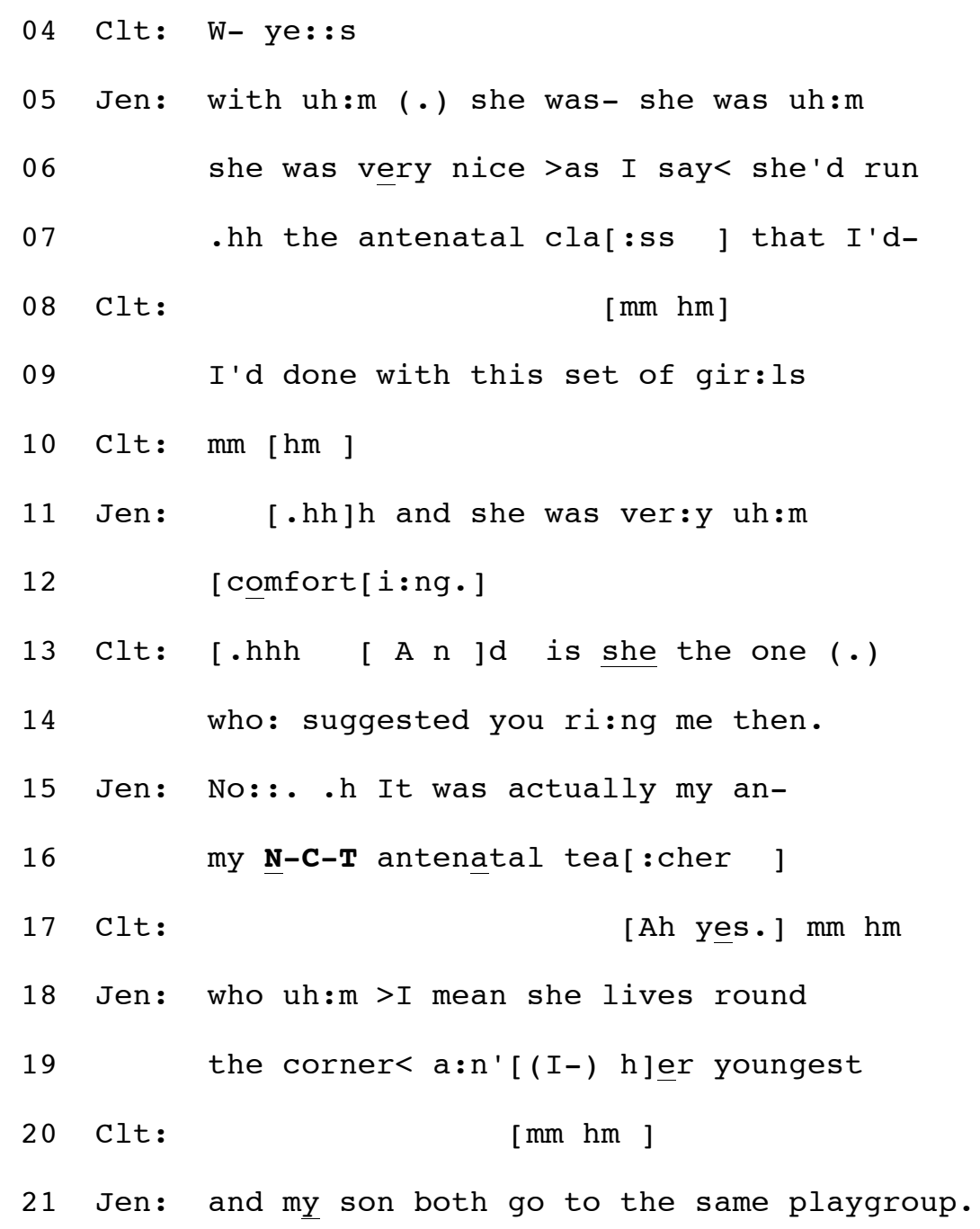

The locally-active alternative referent to my NCT antenatal teacher is one of the ladies who'd run my NHS antenatal class - a different antenatal teacher who has been mentioned some 22 minutes earlier and then made the topic of the talk with a string of locally-subsequent references (she, her). ${ }^{\text {xiii }}$ The insertion repair is part of Jennifer's answer to the call-taker's question (at lines 13-14) as to whether the midwife about whom she is talking is the person who had advised her to call the crisis line. As it happens, it wasn't her just-mentioned midwife - who had also run the antenatal class (lines 6-7), and who could therefore (rather confusingly) be the referent of my antenatal teacher; rather, it was a different antenatal teacher (mentioned earlier, see footnote 10), who had also run a class that she'd attended. In the course of answering, Jennifer hears that the reference formulation she first selected, $m y$ 
antenatal teacher, is vulnerable to being heard as referring to the midwife who'd run the antenatal class (about whom she was speaking and about whom the call-taker had asked her question). She cuts off my antenatal teacher in the course of its production (my an-, line 15) to insert the initialization NCT, identifying the referent more specifically as my NCT antenatal teacher (line 12), and retaining antenatal teacher as part of the repair solution. The insertion of $N C T$ specifies the referent in a way that differentiates between two different antenatal teachers (both of whose classes Jennifer had attended), thereby pre-empting a possible misunderstanding. Here, the insertion of NCT differentiates one person from another, as the insertion of Cary differentiates one place from another.

Immediately prior to the beginning of Extract 23 (from another call to the birth crisis helpline), the caller, Bess, has claimed that the medical professionals supervising her birth agreed (at least in principle) to remove the drip and monitor with which she was fitted during labour so that she could use a birthing pool. (This - rather unusual - procedure is indexed by the call-taker's that in line 2.) The insertion repair is at lines 5-6 where Bess says it was actually my mid- my community midwife - cutting off the production of midwife to insert community, thereby specifying the referent as my community midwife.

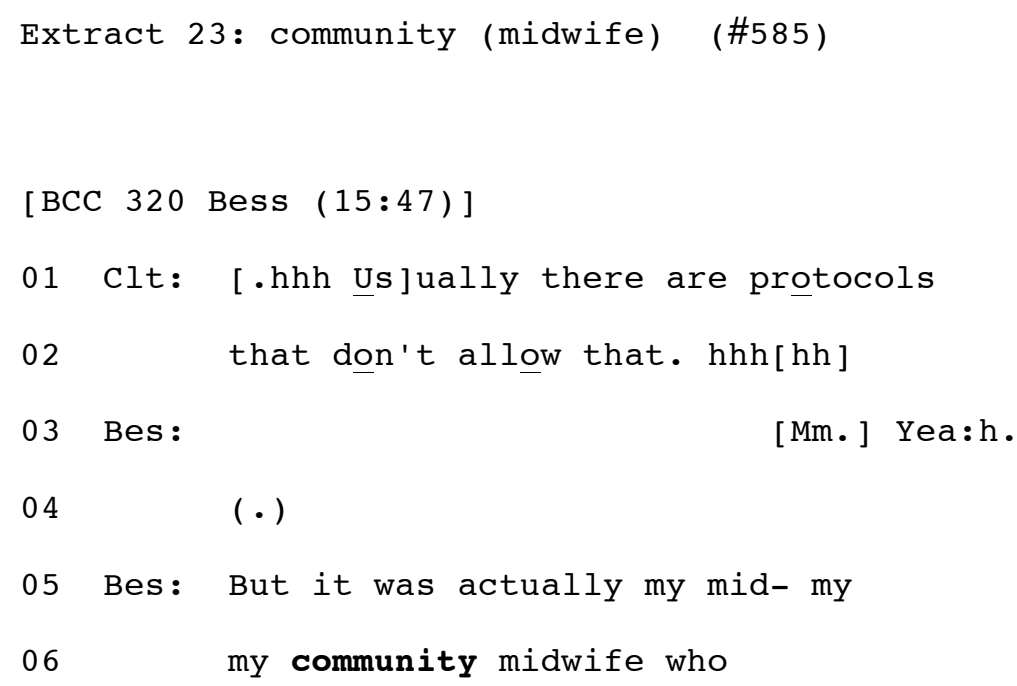


The locally-active alternative referent to my community midwife is the person earlier referred to (see data in footnote) as the midwife in hospital - and this earlier reference was itself produced as the outcome of a repair operation. ${ }^{\text {xiv }}$. In the course of accounting for the medical professionals' (purported) agreement to this unusual procedure, attributing its suggestion to someone with more medical authority than herself (lines 5-7), Bess hears that the reference formulation she first selected, my midwife, is vulnerable to being heard as referring to a different midwife - one to whom she has earlier referred (not in the data extract shown) as the midwife in hospital. The insertion of community modifies my midwife, specifying the referent in a way that differentiates between two different midwives. This differentiation turns out to be critical to the next part of Beth's telling (lines 9-11), which features both midwives.

In Extract 24 the repaired reference formulation has an object (rather than a place or a person) as its referent: a clothes wash. A caller to the (New Zealand) Electricity and Gas Complaints Commissioner's Office is complaining that the electricity company is not supplying enough power to heat her bathwater. Her argument is that, given her thrifty and environmentallyfriendly use of cold water rather than hot for many domestic purposes (washing dishes and washing clothes), there should certainly be sufficient for a hot bath. The insertion repair is at lines 23-24 where the caller, CAL, says I don- uh only (.) EVer do a cold water .hhh wa:sh: $d$ uh di-uh:m (.) clothes wash. There is some initial trouble: di- at line 24 is the beginning of the wrong word, dish, which is replaced by clothes. Clothes is inserted to modify the 
compound reference formulation a cold water wash, thereby specifying the referent as a cold water clothes wash (i.e. she runs her washing machine on cold fill only).

Extract 24: clothes (wash) (\#247)

[EGC Mary10]

01 CON: Righty ho. What can I do for you Maureen.

02 CAL: .hh We:ll ((clears throat)) there's only the

03 two of us in the hou:se

04 CON : Yé[p.]

05 CAL: [.h]h We u:se ((swallows)) co:ld wa:ter in

06 our dishwa:sher .hh we u:se co:ld water in our

07 washing machi:ne .hh we <shas:re a ba:th>

08 every morning and by the time we'd put two

09 inches of water in the bath this morning it

10 was running co:ld. .hh And this (.) isn't good

11 enough for over eighties.hh

12 CON : No: !

13 CAL: [ N $0:$. ]

14 CON: [It isn't.] You need [some] hot wa:ter.

15 CAL : $\quad[\mathrm{N}:-]$

$16 \mathrm{CON}:(0.2)$

16 CAL: Well we: do:.

18 CON : Yea: $\mathrm{h}$.

$19 \quad(\cdot)$

20 CAL: .hh [An' I me]an we:'d we economise by >as I say<=

21 CON: [ Uh:m]

22 CAL: =using cold water in the dishwasher cold wa- I

23 don- uh only (.) Ever do a cold water .hhh wä:sh:

24 d- uh di- uh:m (.) clothes wash

25 CON : Ye[p. ] 


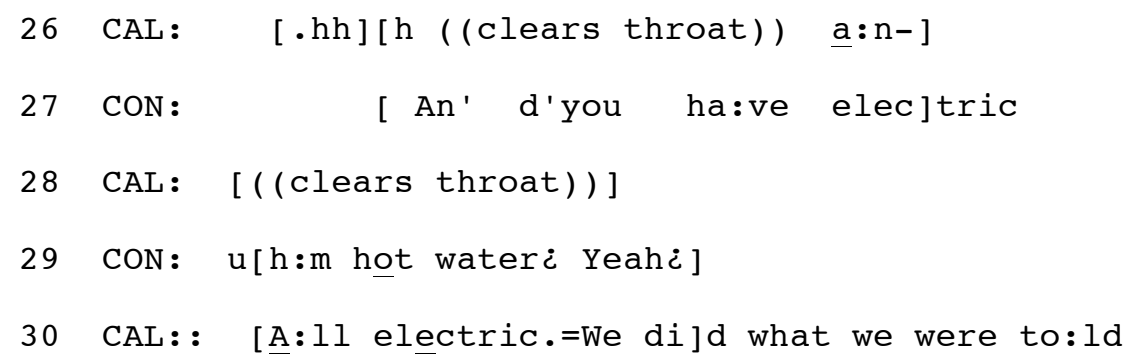

Here there are two locally-active alternative referents. The insertion of clothes (at line 24) modifies cold water wash, specifying it in a way that differentiates it from two other kinds of (cold water) washes to which the speaker has referred in the immediately preceding talk: the washing of dishes (in her dishwasher, lines 6 and 22) and the washing of bodies (in her bath lines 7 and 9). As before, then, insertion repair is used to differentiate between possible referents, thereby pre-empting misunderstanding.

In Extract 25 the repaired reference formulation again refers to an object - a baby's shoulder. A caller to the birth crisis line, Jasmine, is describing a difficult forceps birth involving a stuck shoulder. The insertion repair is at lines 3 and 5 where Jasmine says her sh:- her othher shoulder that wasn't stuck. Here the speaker starts to insert the word other to modify her shoulder but abandons this, and the repair segment cascades (see Lerner, Kitzinger and Raymond, 2009) into a third attempt to specify the referent. The successful formulation is the reformatted version her shoulder that wasn't stuck.

Extract 25: other (shoulder) (\#567)

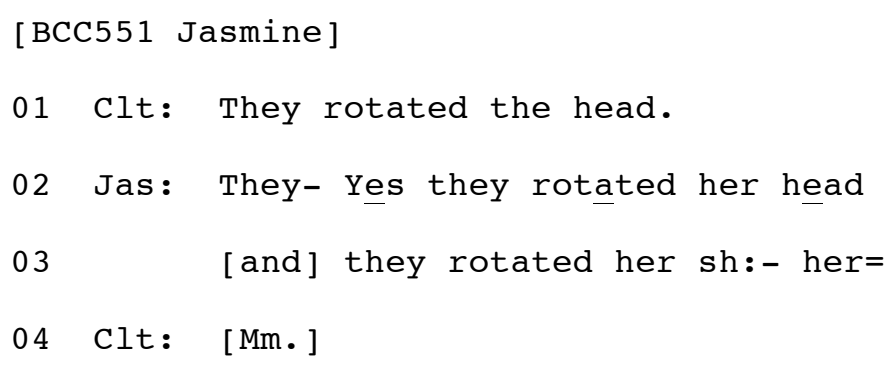




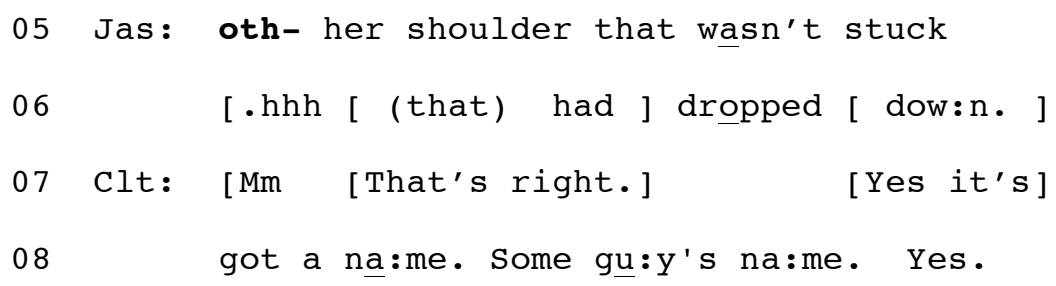

Here again there is a locally-occurring source of possible misunderstanding. The locallyactive alternative referent to her other shoulder (or her shoulder that wasn't stuck) is her shoulder, referred to less than a minute earlier (Jasmine says: basically what happened was she got her sh:oulder stuck under my pe:lvic bo:ne).

Here, then, we have seen how speakers can repair a reference formulation, inserting material which specifies the referent - and this specification is analyzably relevant to differentiating between two (or more) locally-active possible referents (places, people, objects), thereby preempting possible misunderstanding.

\section{(b) Specifying when there is no possible (locally-occurring) alternative referent, in the service of the interactional task-at-hand}

Sometimes there are no such locally-active alternative possible referents. Rather than dealing with a problem of understanding, specification of the referent is designed, instead, to alert the recipient to the relevance for the action of the turn-in-progress (whatever that action is) of the referent being this type or this one: a particular type of chap (26), a particular brand of shoes (28), a particular sort of pain (30). In these instances, then, insertion repair is not just repair although it is also repair. It is analyzably designed to modify a reference formulation so as to make it more fitted than was the original version for the particular interactional task-at-hand: tasks such as credentialing a practitioner (26), accounting for a state of affairs (27) or for an assessment (28), providing evidence for an assertion (29), and setting up a contrast (30). 
When specifying is used to differentiate two referents, it is only a repairing action. When it is used without any apparent problem of differentiation, the insertion repair is being used in the service of the interactional task-at-hand. The repairing action is specifying, but the interactional action is case-specific, and can be - as we will see - anything from credentialing to accounting to providing evidence.

In Extract 26, the speaker, Pam, reports an improvement in her mental health problems, attributing this improvement to the practitioner who has been treating her. The insertion repair is at lines 9-10 where Pam refers to this chap this Islamic chap. She treats the reference formulation she first selected (this chap) as insufficiently precise and - without using an explicit repair initiation (as discussed in the Technology section) - she inserts the defining adjective Islamic to modify her original reference formulation, thereby specifying the referent as this Islamic chap.

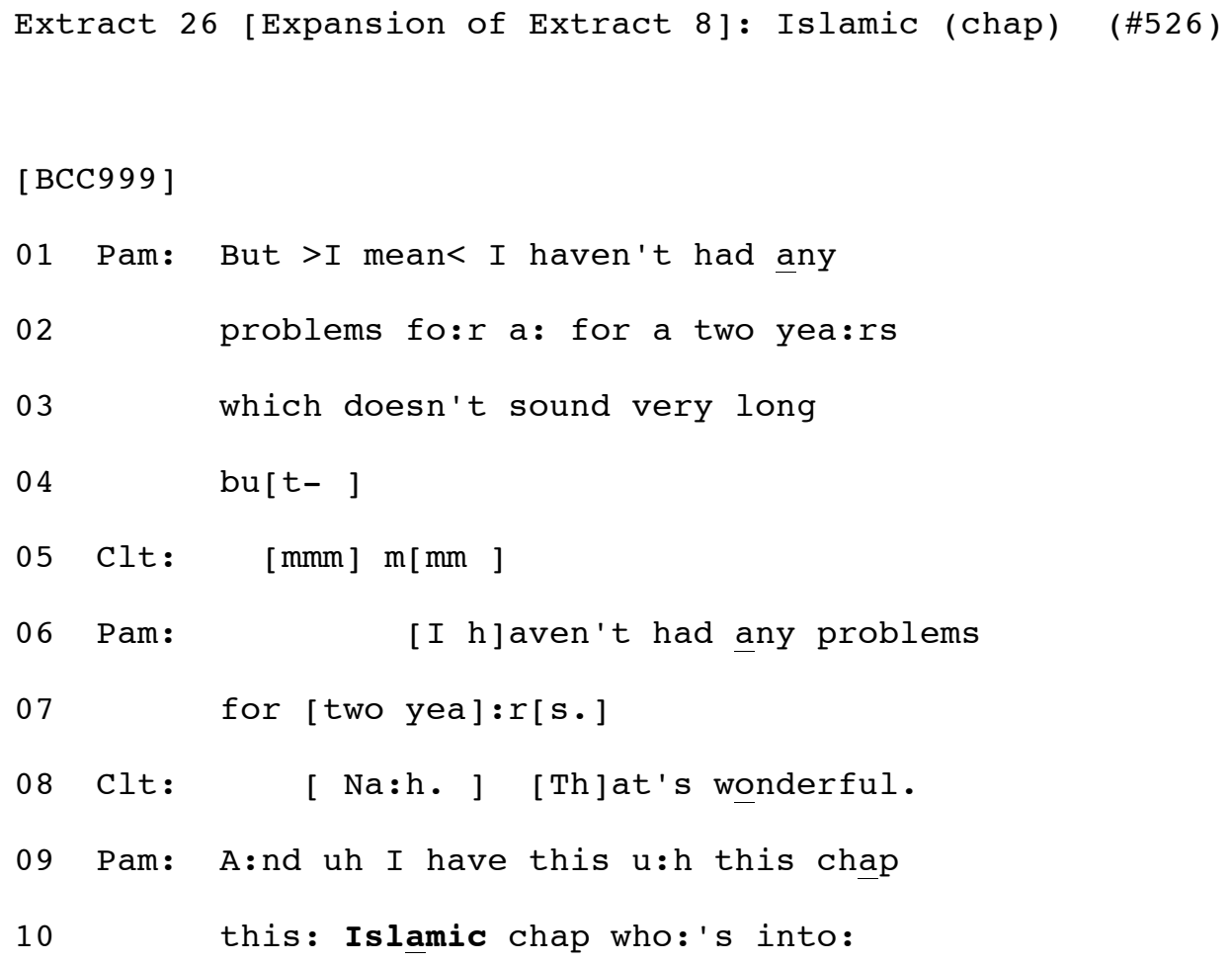




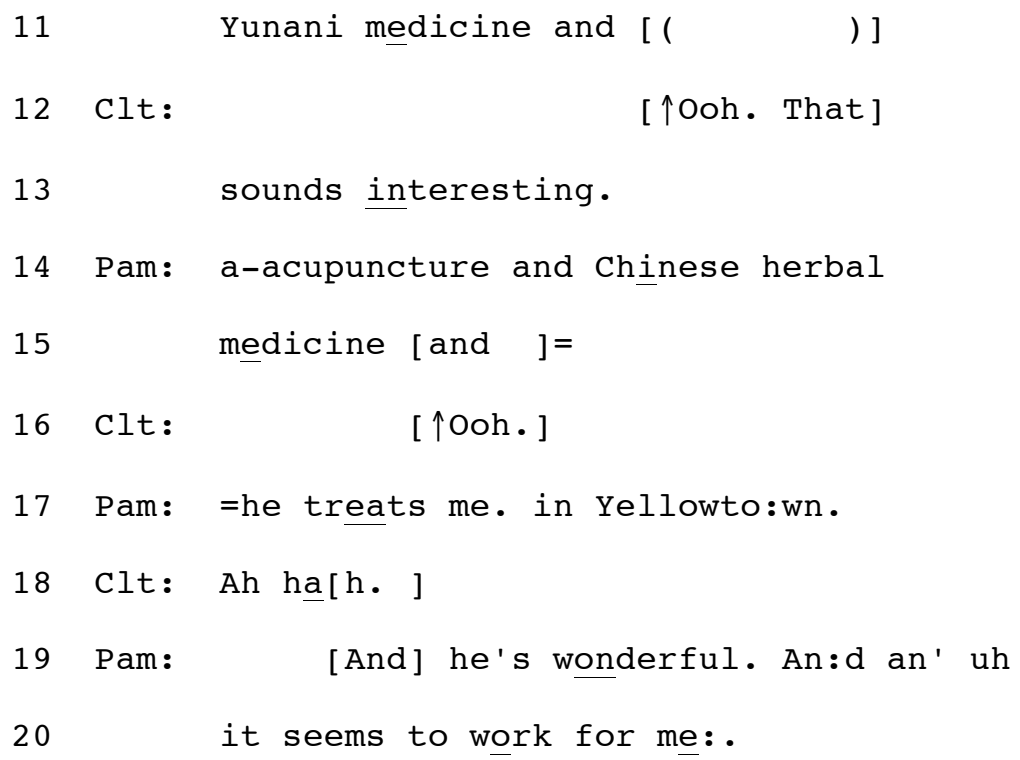

Here there are no locally-alternative referents. No other chap has been mentioned (or is possibly inferable), and the problem is not one of differentiating between two or more possible chaps. Rather, the speaker's specification of chap displays that she is treating the type of chap he is as relevant. The repair solution invites the recipient to figure out exactly how his being this kind of chap in particular - i.e. Islamic - is relevant to the interactional task for which the repair is mobilized. Since Yunani medicine (a branch of medicine popular in Asia) is derived in part from ancient Islamic medicine, it seems that the speaker's specification of the chap as an Islamic chap is done in the service of credentialing him as a practitioner $^{\mathrm{xv}}$.

In Extract 27, a premature baby tethered to machinery in an incubator is described (at lines 45) as having pulled the tube the feeding tube out of his nose, inserting the defining adjective feeding. The baby's grandmother is calling the birth crisis line to report that her daughter is now home (line 1) and breastfeeding her premature baby (line 3). This is treated by both conversationalists as excellent news, since it can be difficult to breastfeed premature babies 
who may not have developed a suck reflex. The insertion repair is part of an account for how this has been achieved.

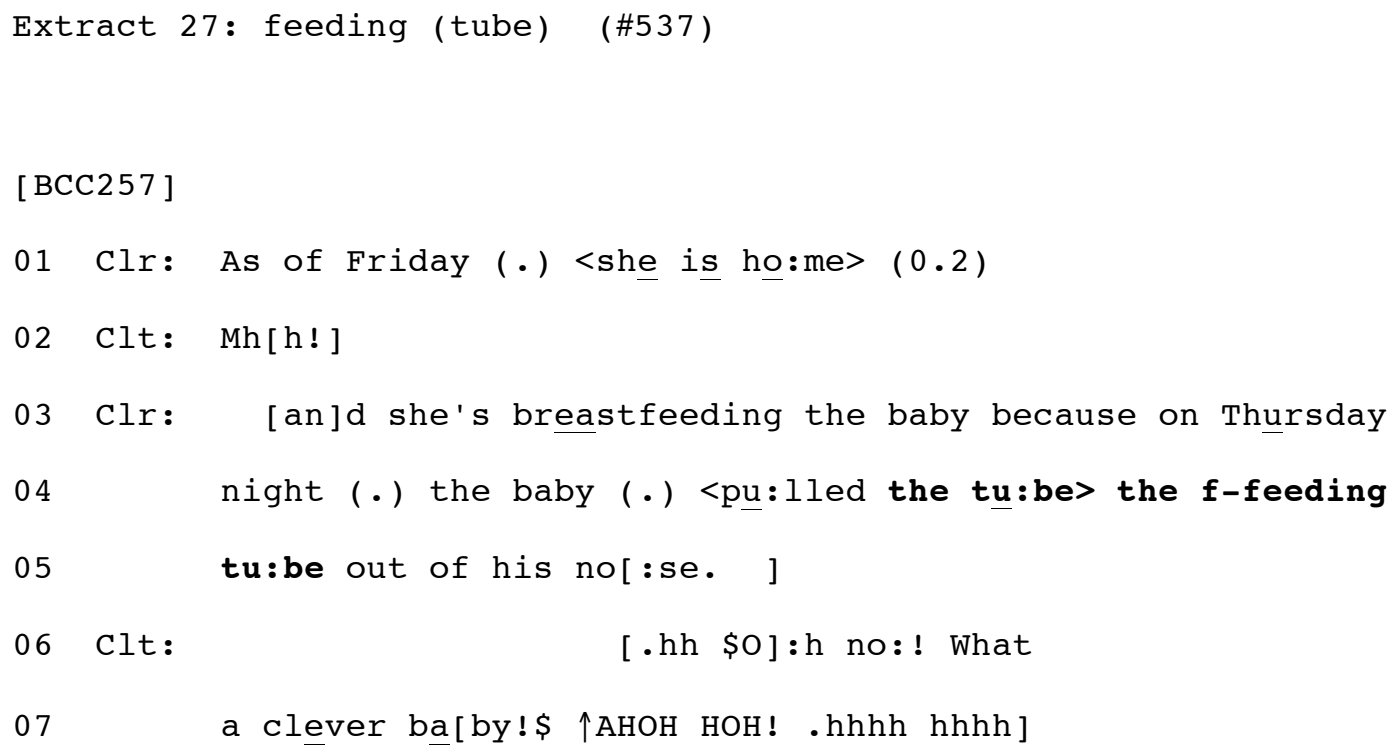

Here again, there are no locally-alternative referents and the problem is not one of differentiating between referents, since no other tube has been mentioned. Rather, the speaker's specification of the tube as a feeding tube displays that she is treating the type of tube it is as relevant, and is inviting her recipient to figure out exactly how it is relevant. The speaker's account attributes agency to the baby (who pulled the tube [out]) - and the account is being offered in the service of explaining how it has come about that he is being breastfed. The specification of the tube he pulled out as the feeding tube in particular treats the baby's action as signalling refusal of artificial nutrition and a demand for the breast. The recipient hears it that way and celebrates the baby's achievement: What a clever baby! (lines 6-7).

In Extract 28, a teenager (DR) is telling a much older friend (IV) about the dangers of walking down a particular street (Cuba Mall) in Wellington, New Zealand. He reports that Cuba Mall 
is frequented by street gangs who steal your bloody sh: Doc Martens shoes (line 15), inserting the well known brand name Doc Martens.

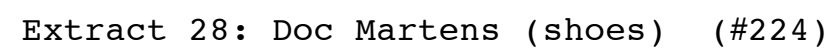


No other shoes have been mentioned, so here again, there is no problem of differentiating between referents. Rather, the speaker's specification of the shoes as Doc Martens displays that he is treating their brand as relevant - and is inviting his recipient to figure out what that relevance is. The repair is part of a turn accounting for the speaker's assessment that the situation in Cuba Mall is pretty bad (line 6), citing widespread street gang thefts in the area as evidence. Since Doc Martens is a well known brand name, it seems that the speaker's specification is done in the service of proposing that thefts in Cuba Mall are motivated by the desire for designer-label goods, rather than a need for basic commodities. This analysis is supported by his subsequent joke (lines 23-24) that the gangs will not steal the corolla - a family saloon car unlikely to be attractive to people with designer goods aspirations.

Extract 29 is from a programme in a radio series offering 'a witty but thought-provoking look' at traditional British institutions: asking, on this occasion, 'What's the point of ... the British Zoo?'. Following an introduction (lines 1-9) from the presenter, Quentin Letts, the programme moves into a pre-recorded interview with the Director of Conservation at London Zoo, David Field, outside the gorilla enclosure. David is extolling the value of the enclosure for rehabilitating badly-behaved gorillas. ${ }^{\text {xvi }}$ The insertion repair is at lines $14-15$ where David refers to the whole different habitats microhabitats in the enclosure, inserting - without explicit repair initiation - the prefix micro, thereby specifying the referent as microhabitats.

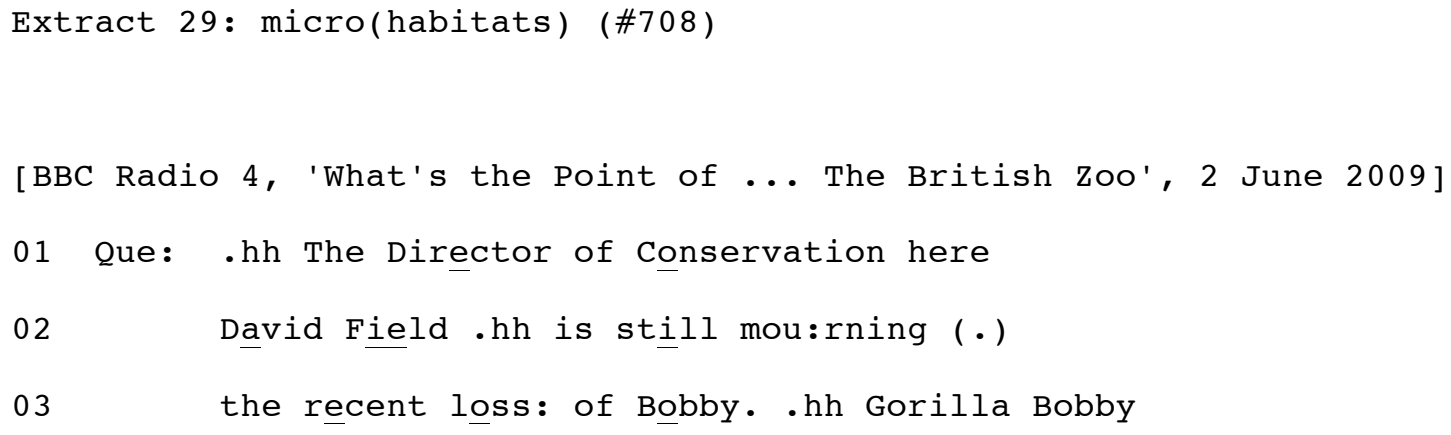




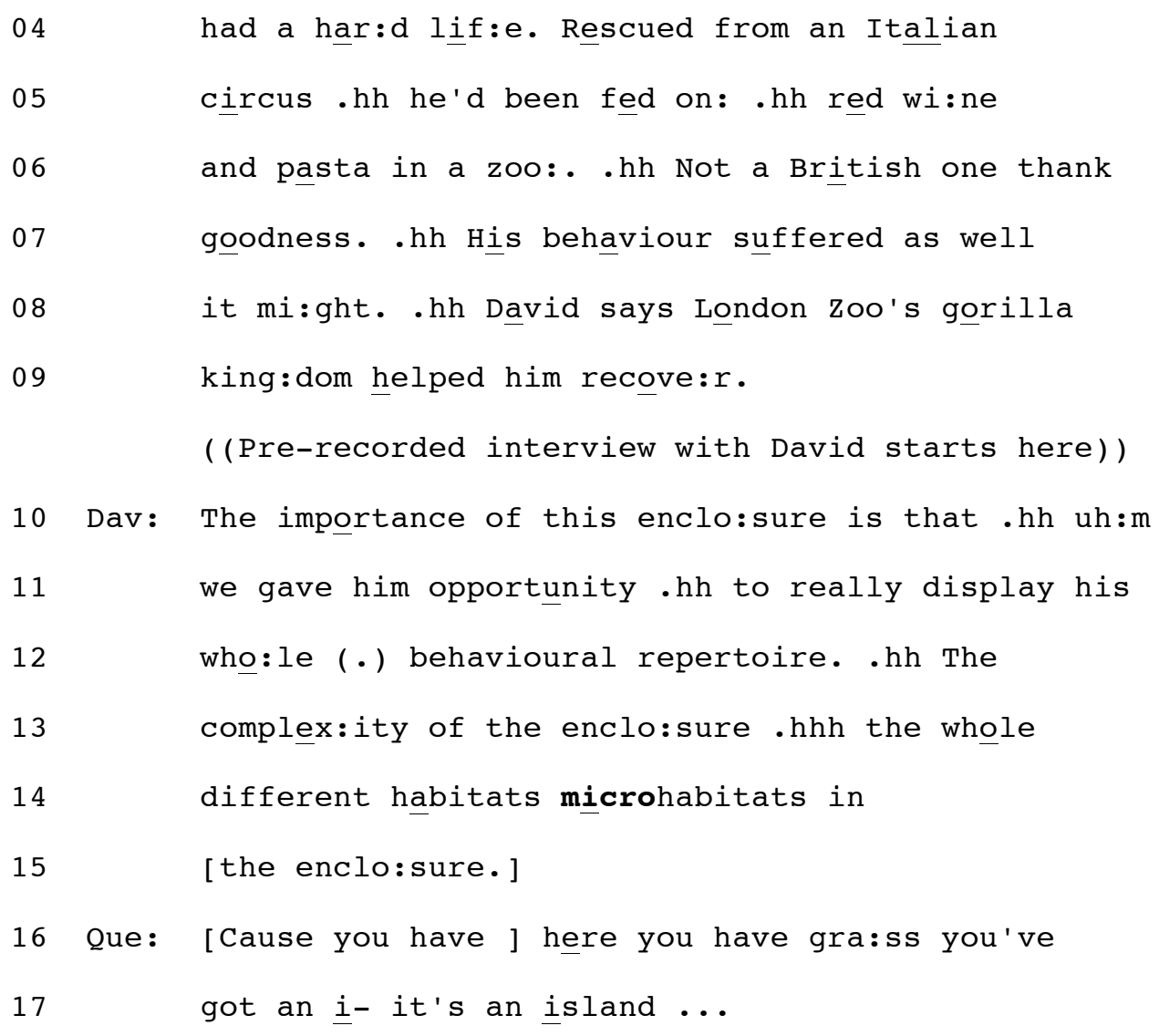

Since no other habitats have been referred to, there are no locally-alternative referents and therefore no possible problem of differentiation. Rather, the speaker's specification of habitats treats the kind of habitats they are as relevant to the interactional task-at-hand. The repair is part of a turn in which the speaker is claiming the excellence of one of his zoo's facilities, thereby defending the zoo in the context of an interview recorded for a programme interrogating the zoo as an institution. He makes a strong claim that the facility was important in offering Bobby the gorilla (sadly now deceased) the opportunity to do everything he might have done in the wild (his whole behavioural repertoire, lines 11-12, is an extreme case formulation [Pomerantz, 1986]), thereby helping his rehabilitation. In support of this claim, the speaker refers to the complexity of the enclosure and then begins to describe how it is divided into different areas, using the reference formulation habitats to convey something of the 'naturalness' of the environment provided, and then repairing habitats to microhabitats to convey the existence of a range of specialised areas within which Bobby could display 
different natural behaviours. The recipient hears it that way and begins to name (presumably, at least in part, for the overhearing radio audience) specific instances of what he can see within the enclosure and takes to be examples of these different microhabitats (grass, an island, lines 16-17). The speaker is using the insertion repair to provide evidence for his assertion that his zoo had helped Bobby the gorilla, thereby defending the zoo.

In Extract 30 Vita - another caller to the birth crisis line - is describing a traumatic birth experience. She says the pain was- the physical pain was not the worst thing (lines 2-3), inserting the defining adjective physical to specify the type of pain to which she is referring.

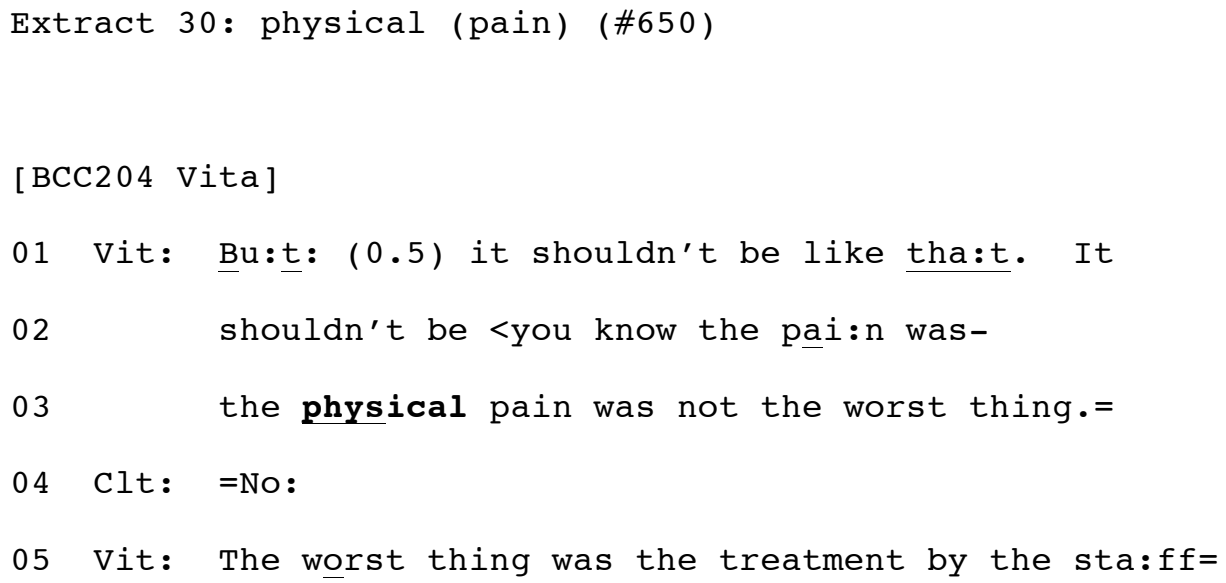

No other kind of pain has been mentioned earlier in this conversation. Moreover, the ordinary understanding of pain is - generally speaking - that it is physical: although pain can refer to severe emotional or mental distress, this is usually a secondary meaning - a kind of metaphorical extension. The relevance of specifying pain as physical pain is not, then, to differentiate it from some alternative type of pain already mentioned, but rather to invoke the existence of emotional pain by naming an alternative to it. The treatment by the staff (line 5) can then be understood as having caused her this kind of pain, without it being explicitly stated. Without the specification of pain, the contrast between not the worst thing (line 3) 
(i.e. the pain, assumed physical) and the worst thing (the treatment by the staff) would not carry the implication that the treatment by the staff caused her pain (of an emotional type). The interactional task-at-hand for this speaker is detailing the extent of her suffering and by invoking emotional as well as physical pain the specification contributes to her account of her birth experience as traumatic and to blaming the hospital staff.

Specification, then, is a key action accomplished by insertion repair. Speakers repair reference formulations, inserting material which specifies the referent more closely or in more detail, and do so analyzably to differentiate between two (or more) locally-alternative referents or in the service of the interactional task-at-hand. ${ }^{\text {xvii }}$

\section{(2) Intensifying:}

In what we are calling intensifying, the inserted material modifies the original formulation so as to strengthen or intensify the meaning of the word(s) it modifies. Insertions can intensify formulations of action (e.g. I wouldn't worry [36]; we're not buying [33]; it may bring [35]); people (a petrified husband [31]); places (a new museum [32]); and objects (an unpleasant text message [34]). The inserted material - often, but not always, an adverb (and often what linguists call an intensifier) - is usually a single word (e.g. really, completely, extremely) that has little meaning in and of itself. In the repaired versions, I wouldn't worry becomes I really wouldn't worry [36]; we're not buying becomes we're absolutely not buying [33]; it may bring becomes it may well bring [35]; the petrified husband becomes completely petrified [31], the new museum becomes spanking new [32] and the unpleasant text message becomes extremely unpleasant [34]. In each case the repair solution is a stronger formulation than is the original. 
Again, the original formulation is treated as not wholly wrong, and so not in need of deleting or replacing. However, unlike insertion repairs doing specifying (which treat the original formulation as insufficiently specific), these intensifying repairs treat it as insufficiently strongly formulated. In many, the repaired version becomes - by virtue of the inserted material - an extreme case formulation (Pomerantz, 1986), as in Extracts 31-33. ${ }^{\text {xviii }}$

In Extract 31, from a helpline call, the speaker (Rita) is describing plans for her forthcoming labour.

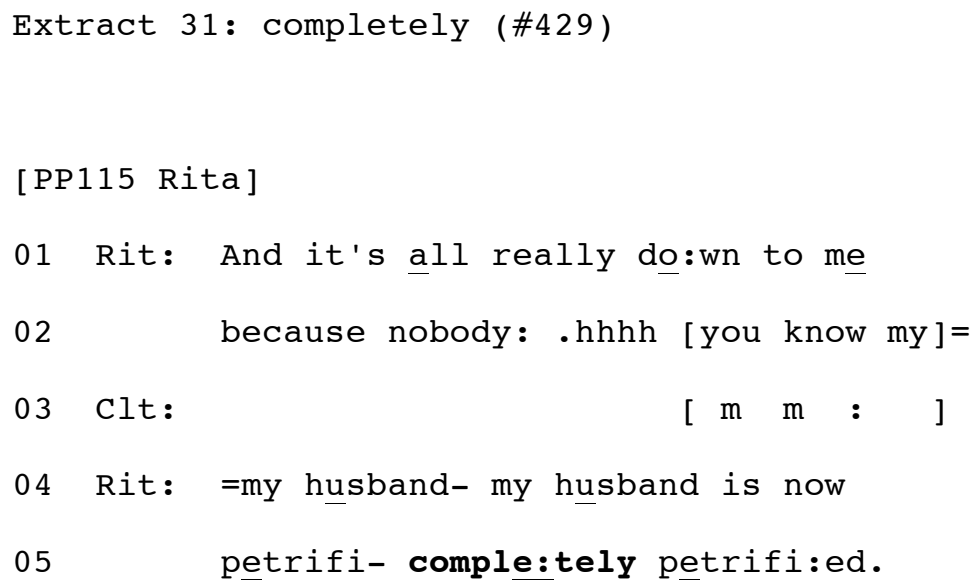

Rita's description of her husband is offered as part of an account for why decisions about labour are her sole responsibility (line 1); inserting completely before petrified intensifies her description of her husband's fear (thereby strengthening her account for his non-involvement).

In Extract 32 the presenter on a radio arts programme is introducing the next item and naming the museum whose pottery collection he is about to describe.

Extract 32: spanking (\#695)

[BBC Radio 4, Front Row, 23 March 2009] 
01 Pre: I'm now at thee ne:w (0.2) spanking new (.) Wedgwood

Inserting spanking before new intensifies the newness of this museum, opened only a few months earlier, and - according to the critics - a state-of-the-art modernist triumph replacing the original 1906 museum. This may serve to highlight the newsworthiness of the report.

Extract 33 comes from a radio news programme discussion about the 2008/9 economic crisis.

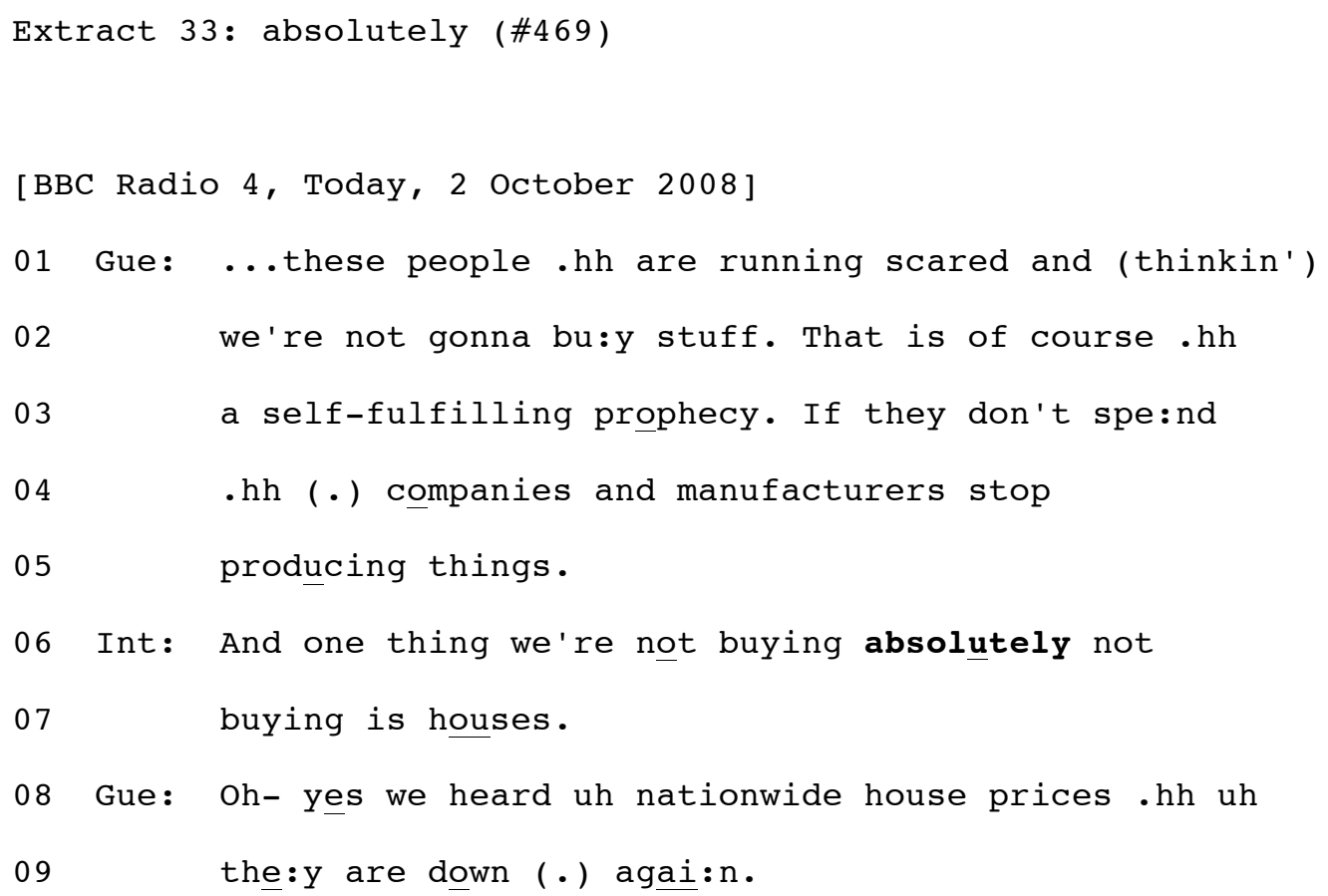

The interviewer is moving the discussion away from manufacturing industries and cueing the studio guest (an economist) to talk about the housing market; he inserts absolutely before not buying, intensifying the public's unwillingness to purchase houses (and thereby increasing the importance of this as the next discussion topic). 
Extract 34 comes from a radio interview with the lawyer representing a lesbian soldier (Kerry Fletcher) who has just won a sexual harassment case and received a large compensation payment from the (British) Ministry of Defence. The interviewer's question (lines 1-2) about what Kerry Fletcher went through is asked in the context of media condemnation of the level of compensation (which, subsequent to this interview, was reduced by a third [The Times, 2009]). The insertion repair is at line 6, where the lawyer says that the harassment began when Fletcher's N-C-O (non-commissioned officer) sent her a number of unp-extremely unpleasant text messages.

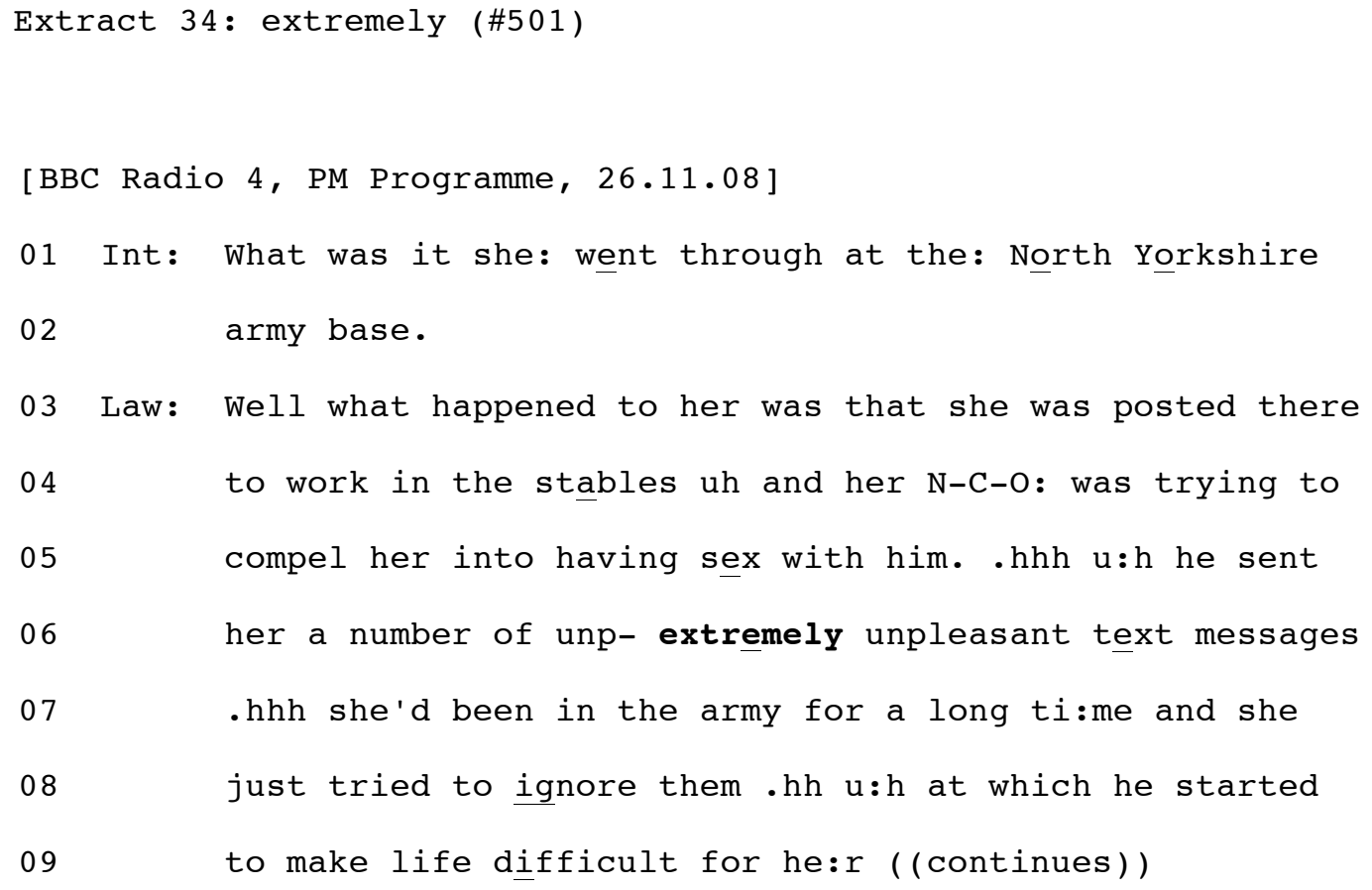

Inserting extremely intensifies the unpleasantness of the text messages and hence the severity of the harassment (thereby contributing to the lawyer's ongoing action of justifying the level of compensation his client received).

Extract 35 comes from an out-of-hours call to the doctor, in which there is considerable misalignment between caller and doctor (Drew, 2006). The caller is worried about her two- 
year-old daughter who has a "terribly unbelievably hot temperature" (data not shown); the doctor has discovered (from the caller's responses to his diagnostic questions) that this high temperature started only recently and that the child has been given Calpol (a paediatric analgesic containing paracetamol) just half an hour ago, such that it has not yet had time to work. The insertion repair is at line 4, where the doctor says It may bring (.) may well bring the temperature down.

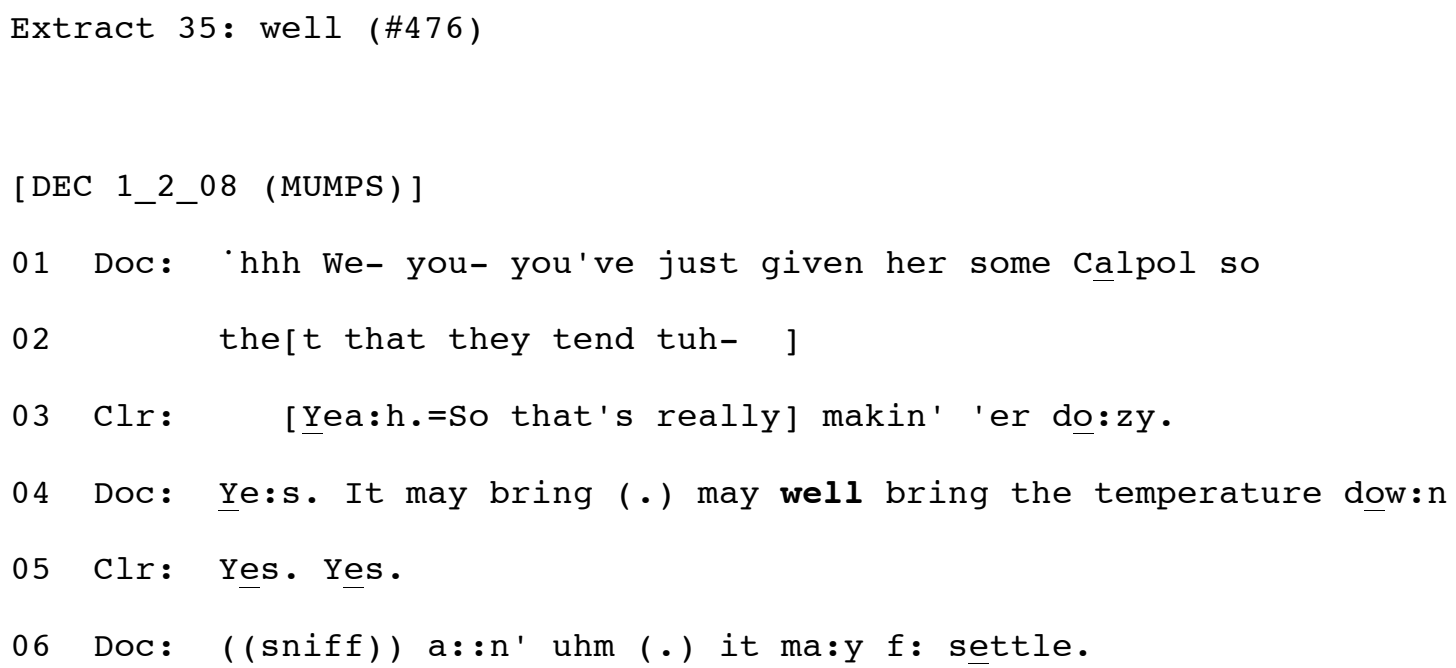

Inserting well intensifies the doctor's claim that the medication will be effective (thereby contributing to his reassurance of the worried mother).

Compared with the wide range of material that can be inserted to do specifying, the set of terms used for intensifying is far more limited. The words really, very, just ${ }^{\mathrm{xix}}$, quite and much account for around three-quarters of all the instances of intensifying in our collection. The single most common word (across UK, US and NZ English) is really, as in Extracts 36-38 below.

Extract 36: really (\#624) 


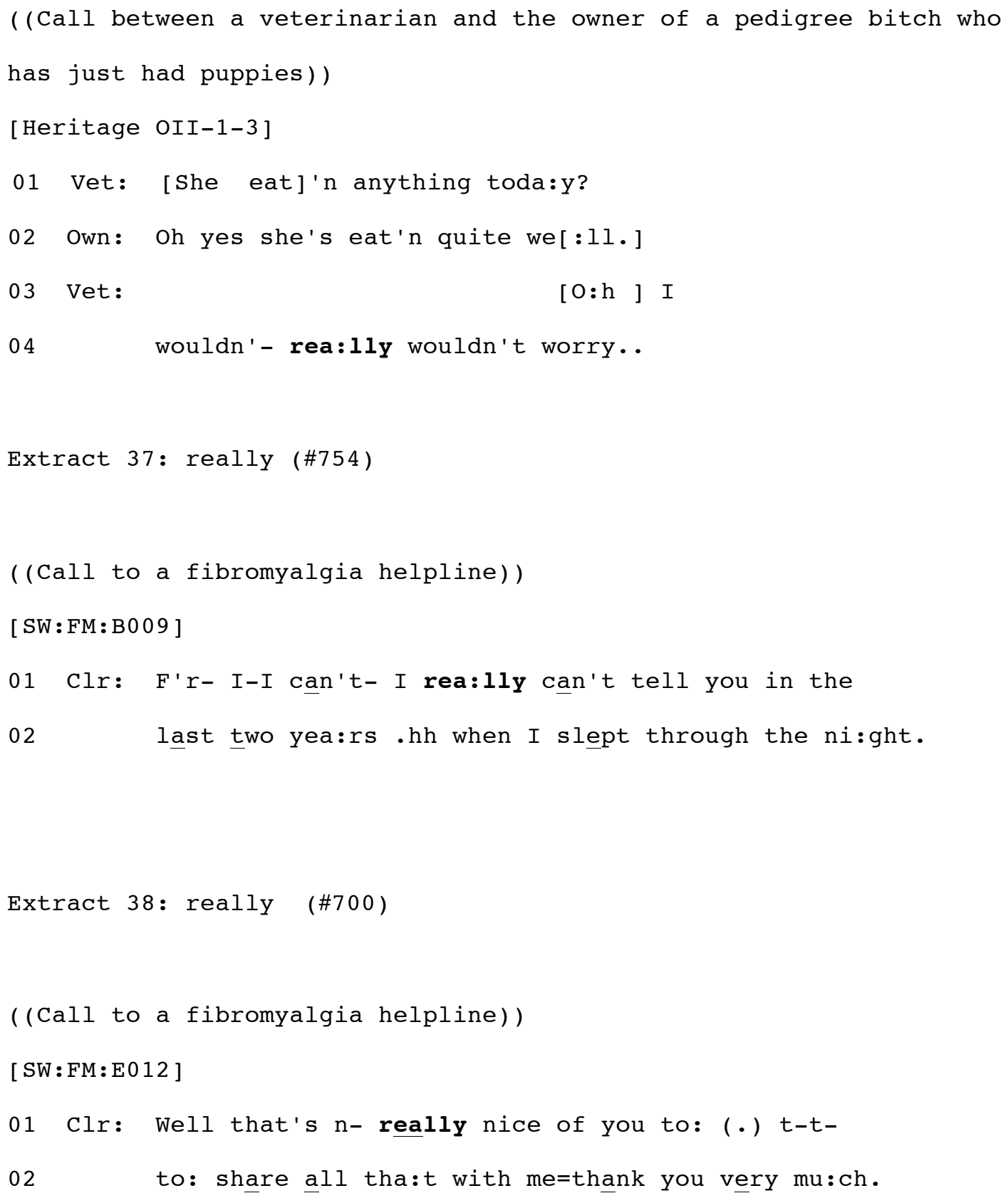

Adverbs - specifically intensifiers - are grammatically dedicated to doing the work needed for intensifying, and so, not surprisingly, are very frequently inserted for this purpose. Other kinds of grammatical objects can, however, also be used to do the same work. In Extracts 39 and 40, speakers insert (respectively) the prepositional phrases on earth and in the world - in each case producing an idiomatic expression which intensifies the original formulation.

Extract 39: on earth (\#627) 


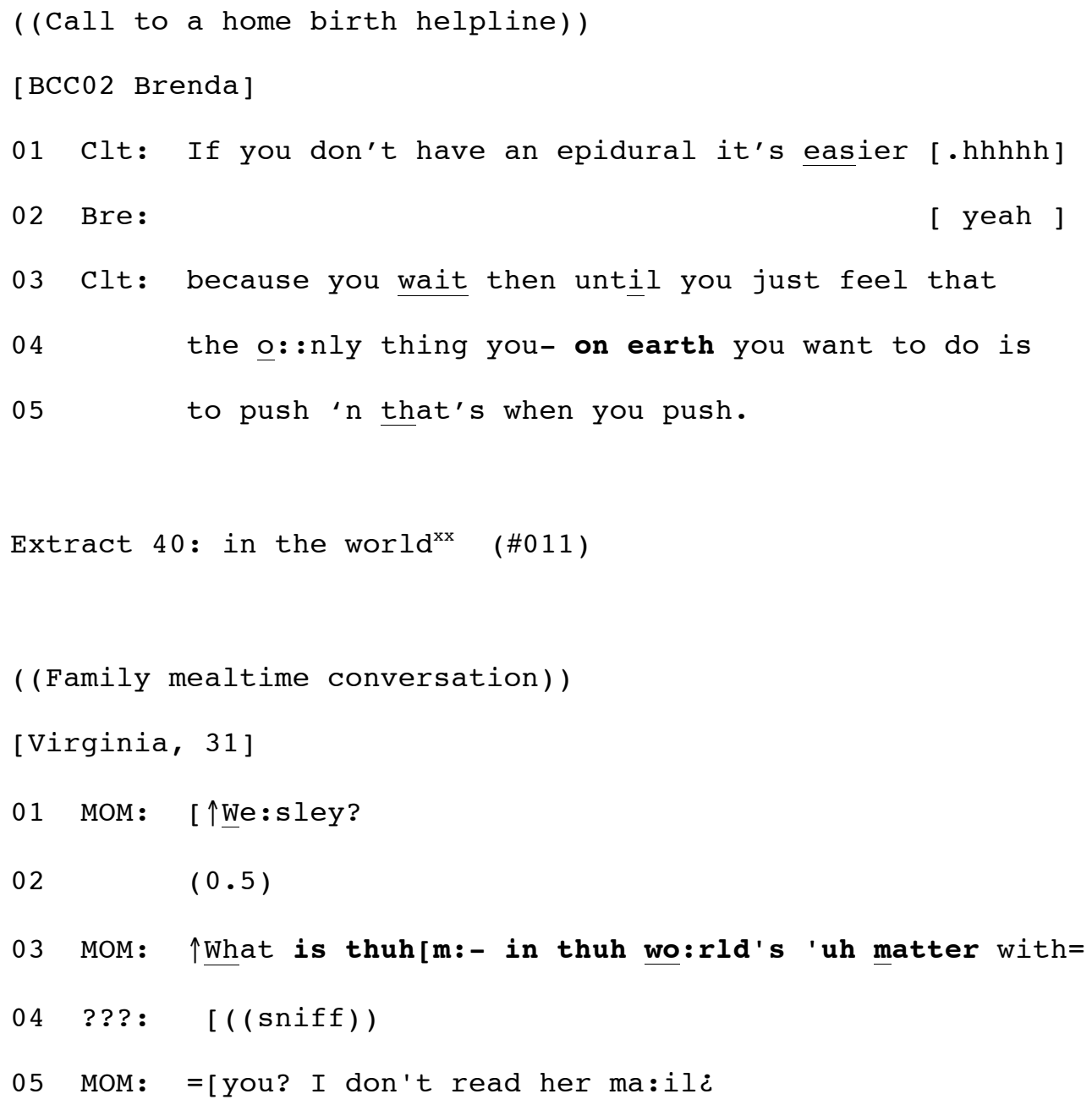

What Extracts 31-40 show is a set of insertions which, as a class, are doing the repairing action of intensifying. These extracts display instances in which speakers are halting the onward progressivity of their talk in order to do insertion repairs analyzably designed to intensify the original formulation. The interactional relevance of these insertions is casespecific (and space precludes detailed analysis). The point of the speaker's doing intensifying, and the recipient's understanding of it, requires further analysis on a case-by-case basis.

\section{(3) Other Actions:}


The two actions already described - specifying and intensifying - together account for more than three-quarters of our insertion repairs. There are four much less common repairing actions in our collection - describing, correcting, adjusting, and expanding - which together account for the remainder of the collection. These are shown below.

\section{(i) Describing}

Speakers sometimes insert a descriptor (e.g. a descriptive adjective or an adverb of manner) to describe a person, object, place or action, etc.: as in dreadful before fires (Extract 41), long before list (Extract 42) and happily before watching (Extract 43).

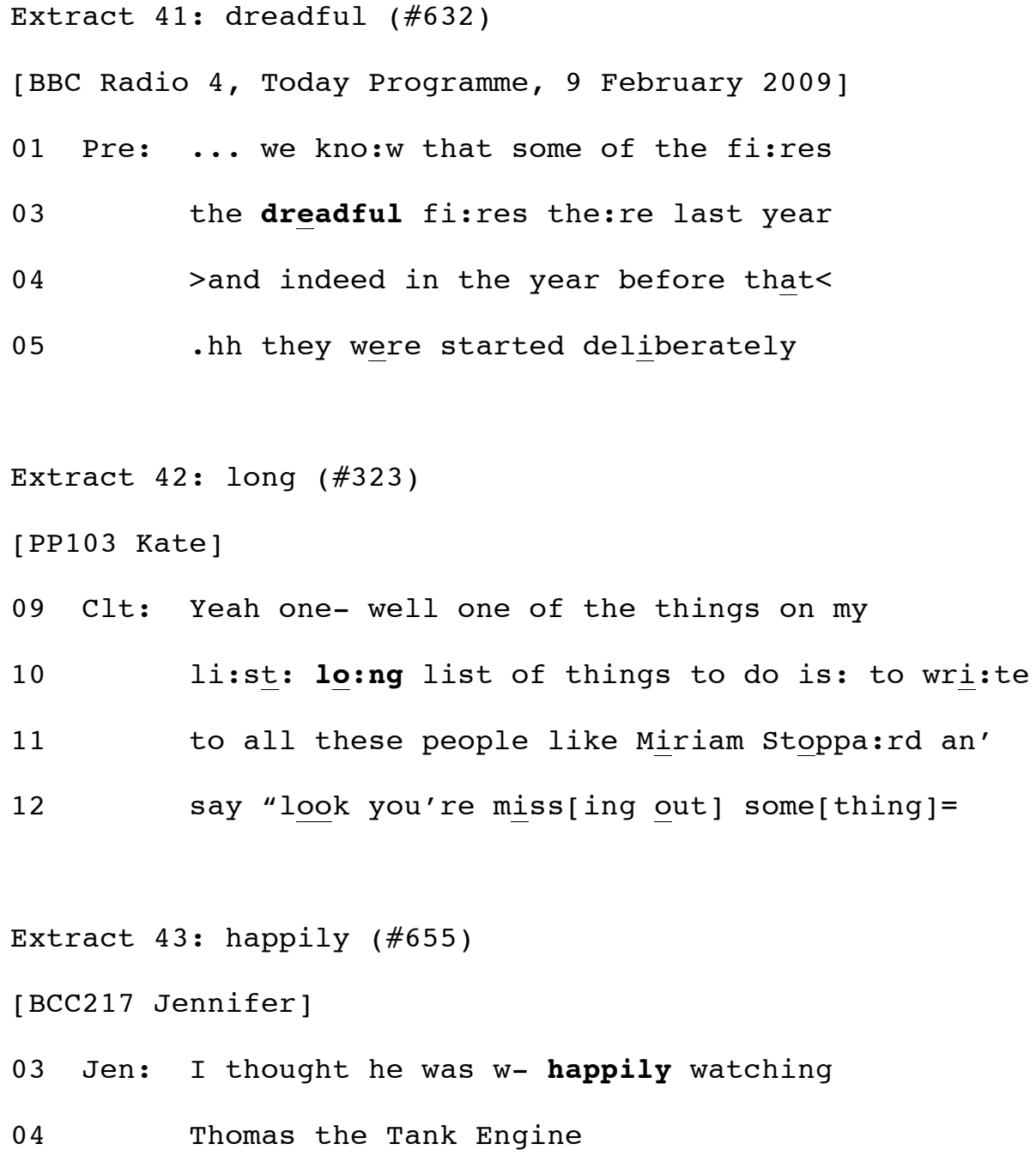


In each case the insertion modifies the formulation by describing (rather than specifying or intensifying) it.

Insertions of descriptors - in particular descriptive adjectives - are relatively rare compared with insertions of defining adjectives. Another way of saying this is that it is much more common for speakers to halt the onward progressivity of their talk in order to specify a referent than to describe it.

When the speaker inserts long before list (in Extract 42), long describes the list (i.e. as lengthy, with many items) rather than defining it as a type of list (as would a longlist preparatory to drawing up a shortlist for a job interview). The issue of what people are doing in describing via insertion repair can only be analysed on a case-by-case basis, and space precludes detailed analysis of this.

\section{(ii) Correcting}

Insertion repair can be used to correct (independently verifiable) errors in talk. In Extract 44, the speaker, Kathy, makes an error in producing the colloquial expression clued up, producing the first part of the word about too soon, in the place when up should come, and cutting it off to insert the missing word.

Extract $44:$ up (\#210)

[ WCSNZE C3A 
01 Kat: ... like men are sort of really clued ab-

In Extract 45, a radio discussion of the book 'Zen and the Art of Motorcycle Maintenance', the guest makes an error in the book's title, producing the first part of the word Motorcycle too soon, in the place when Art of should come, and initiating repair (with an elongated sound part way through) to insert the missing words. He adds an ironic commentary (lines 2-3) on his own error (what Schegloff [2007: 142-8] calls a 'post-completion musing'), eliciting laughter from the interviewer.

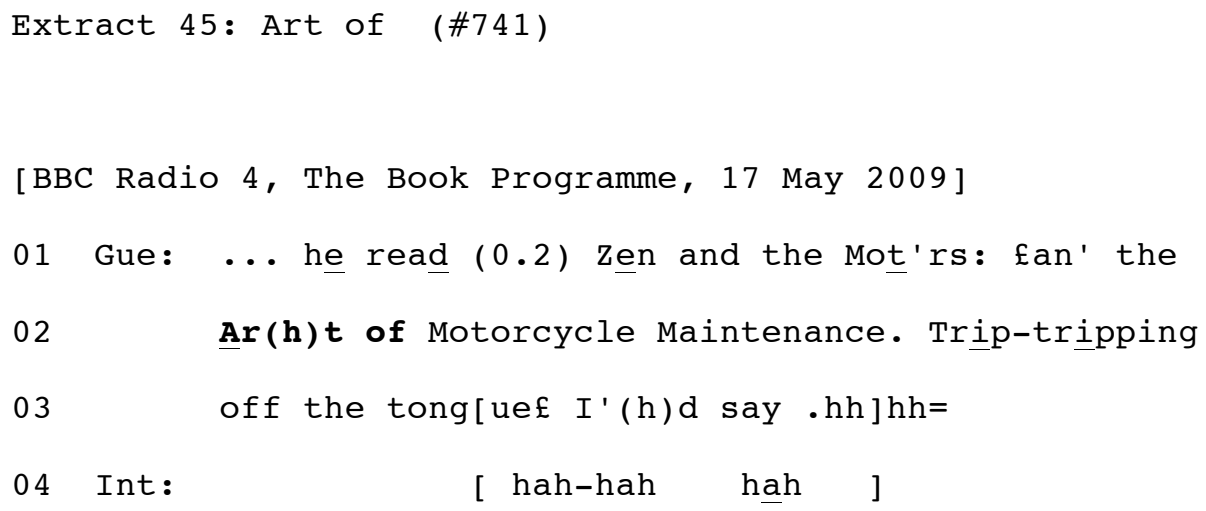

The errors in 44 and 45 are both 'carry-forward errors', i.e. errors of speaking in which a speaker produces (or begins to produce) too soon an item projected to come later in the TCU (Lerner and Kitzinger, 2010). Insertion is, of course, the repair operation dedicated to fixing carry-forward errors, since what is required to correct them is the insertion of material that should have been there on the first saying.

There is a sense in which these corrections, in particular (and also some instances in the other classes of insertions) can give the impression of being a replacement, particularly when the insertion modifies the referent in a major way (e.g. the insertion of cotton before wool to produce cotton wool, which is a wholly different substance). However, even if the modified 
formulation has the appearance of a replacement, it is produced through the operation of insertion: i.e. using the same technology as described in the first section of this paper.

\section{(iii) Adjusting}

Because, as we have seen, insertion repairs retain the original formulation as part of the repair solution, they are well-suited to the action of adjusting it. Two common adjustments relate to epistemic formulations (Heritage and Raymond, 2005), and to action formulations.

\section{(a) Adjusting epistemic formulations}

Speakers sometimes use insertion repair to adjust the epistemic status of their claims, thereby displaying an orientation to the state of their own knowledge (or, less often, to that of their recipient). They do this by inserting epistemic markers such as (most commonly) I think (4648; see also Kärkkäinen [2003]), I guess, or I'd say; also possibly, probably (49), obviously $(50)$.

In Extracts 46-48, speakers insert $I$ think to modify assertions (about morning sickness [46], the name of a boy [47], and the price of shoes [48], respectively) - in each case, displaying lesser certainty than that conveyed in their original formulation.

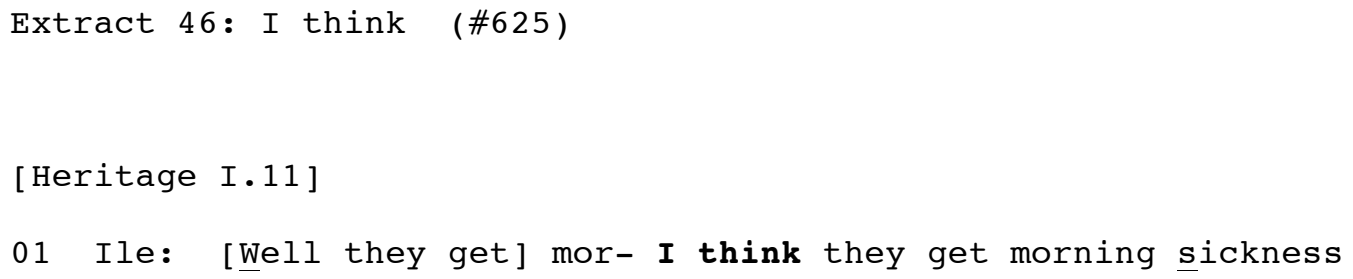




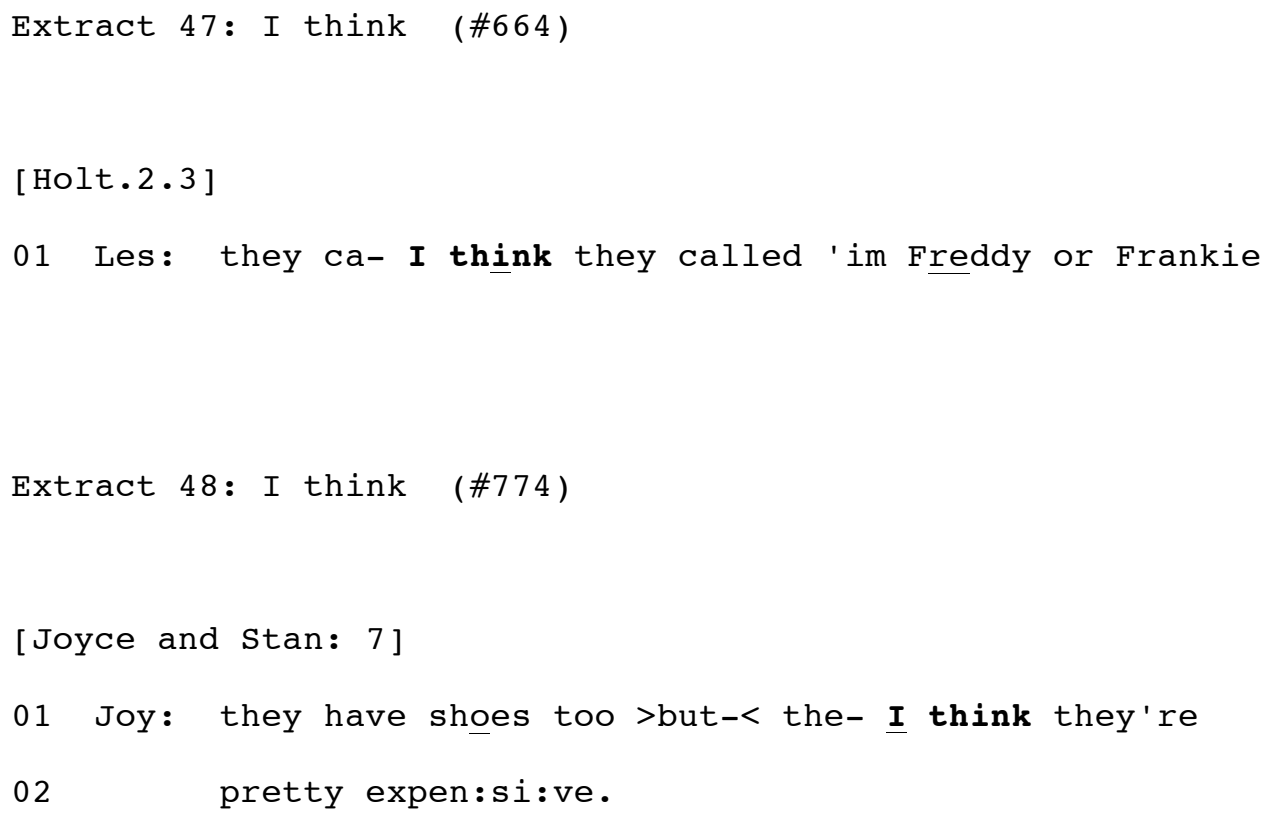

In Extract 49, the speaker's insertion of probably is attentive to the fact that she is making a claim about her recipient's experience (a B-event statement, Labov and Fanshel, 1977) - a domain of knowledge to which the recipient, and not the speaker, can lay claim to epistemic privilege. By inserting probably she displays her orientation to her lack of epistemic authority and draws back from the stronger claim-in-progress that her knowledge about women (line 1) in general necessarily applies to the particular woman who is her current recipient (see also Author 1, 2010).

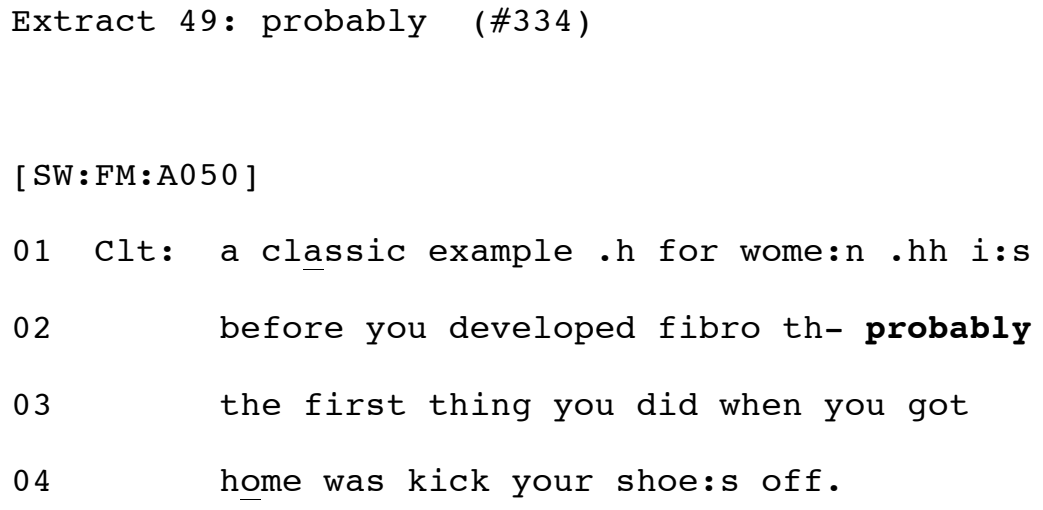


In Extract 50 the speaker is describing her labour and has just recounted how she has been attached to multiple drips, monitors and a foetal probe (data not shown) - all of which would have made it apparent to her recipient that she would not have been able to walk around. She inserts obviously to display her orientation to the fact that in saying I couldn't walk she is telling her recipient (the helpline call-taker) something that she should properly assume her recipient already knows, or should know (a violation of the principle of recipient design, Schegloff, 2007: 38).

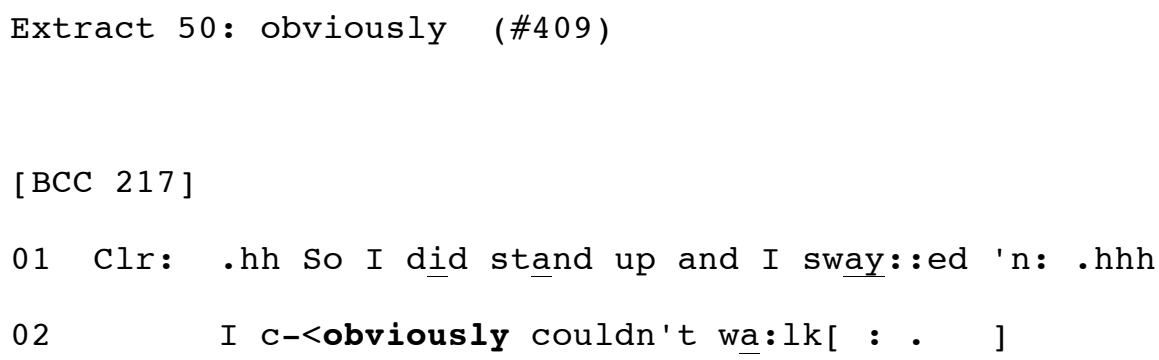

These data extracts (46-50) show, then, that insertion repair can be used to adjust epistemic formulations.

\section{(b) Adjusting action formulations}

Speakers can also use insertion repair to adjust the way in which an action is formulated. In our collection the inserted material is generally another action formulation such as try, managed, decided, had, would (what linguists call an auxiliary or catenative verb) whose insertion results in a TCU with a chain of two - or sometimes more - verbs (e.g. trying to get, managed to get, decided to bring, had to wait, would give), with the newly-inserted verb modifying the main verb and thereby adjusting the action formulation. 
The single most common way in which speakers adjust action formulations is by inserting material designed to display a limited expectation of success and/or an orientation to the possibility of failure. For example, in Extract 51 the speaker inserts trying before her original action formulation (getting together a date for coming down there) to produce the repaired version I'm trying to get together a date. ${ }^{\mathrm{xxi}}$

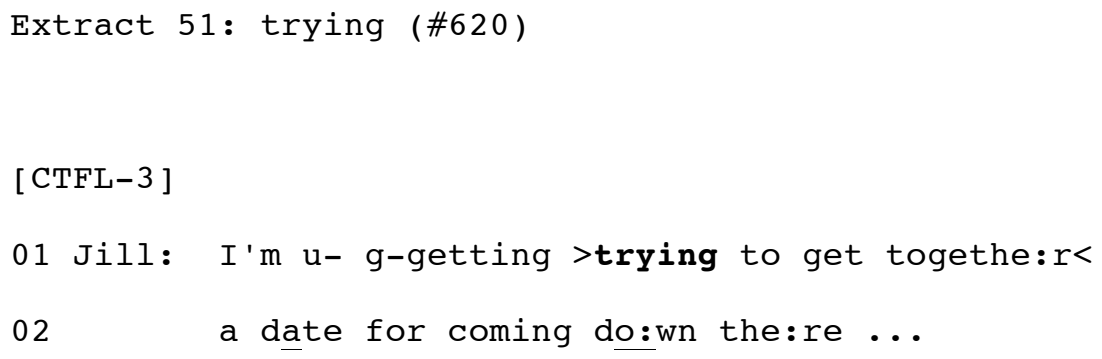

In Extract 52, the speaker inserts try and before the original formulation stay positive (and also amends positive to as positive as you can).

Extract 52: try (\#733)

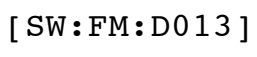

In Extract 53, a valediction for the world-renowned physicist, Stephen Hawking, the speaker (Barack Obama) inserts attempt to before explain - bringing off a joking derogation of his own capacity to understand theoretical physics.

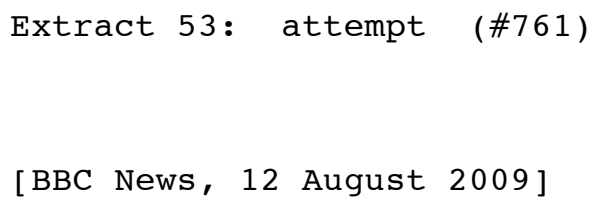


Other adjustments can be made to convey the effort, compulsion, or choice involved in actions. In Extract 57, the speaker inserts managed into her action formulation to convey a sense of the effortfulness involved, repairing I haven't got him off to sleep at all to I haven't managed to get him off to sleep at all.

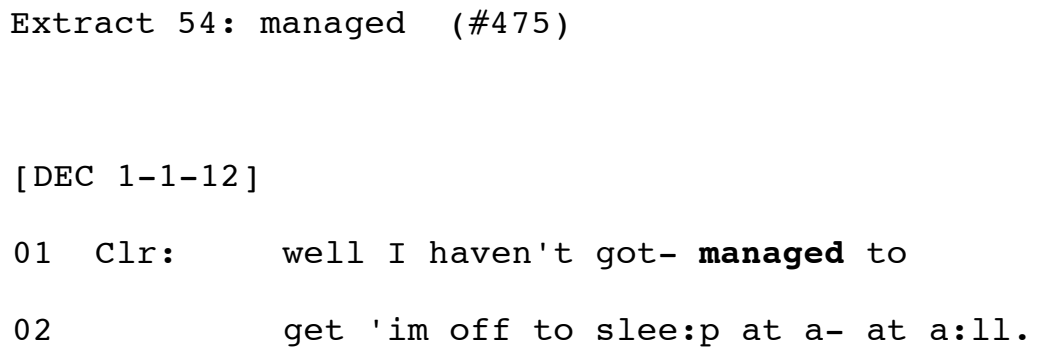

In Extract 55, the action formulation (waited, line 13) is adjusted by the insertion of had (line 14) to render the repaired version had to wait (line 14). This adjustment conveys a sense that the action being formulated was inflicted coercively rather than freely chosen.

Extract 55: had (\#773)

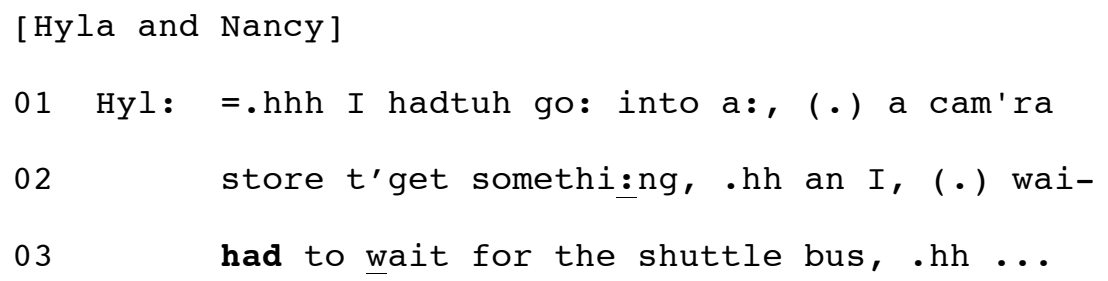


These data extracts show, then, that insertion repair can be used to adjust action formulations.

\section{(iv) Expanding}

In all the insertion repairs we have looked at so far - those which do specifying, intensifying, describing, correcting, and adjusting - the inserted material modifies a formulation. In what we are calling expanding, the inserted material does not modify a formulation that is already there but adds something altogether new. This material is: (a) a new reference formulation additional (or alternative) to one already present in the turn so far; (b) a new formulation of time or place; or (c) one of a large number of other possible (but infrequent) additions.

\section{(a) Expanding with an additional (or alternative) reference formulation}

In Extract 56 the speaker inserts and her hands between the words feet and will in the original version of the TCU. The inserted material does not modify feet (as would, for example, little feet), nor does it modify will (as would, for example, probably will). Instead it adds something altogether new - a reference formulation which the conjunction (and) shows to be an addition (feet and hands). Hands is a reference formulation additional to one already present in the turn (feet) and is analyzably related to it (as a co-class member of the class of body part extremities).

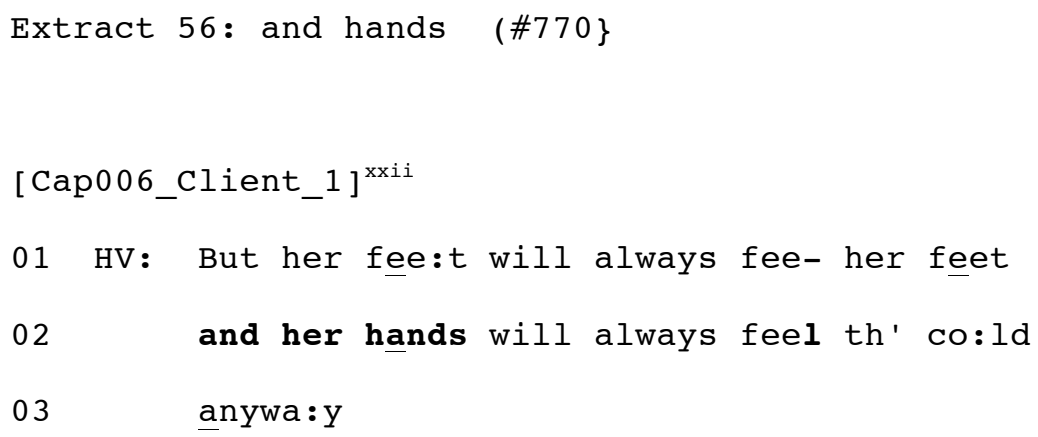


In Extract 57, a speaker inserts and mums between the words newborns and being in the original version of the TCU. Again, the inserted material adds something altogether new, so that the speaker is now referring to both newborns and mums (with the new addition analyzably a member of the same membership categorization device: possibly family, possibly the childbirth pair of newborn and mum).

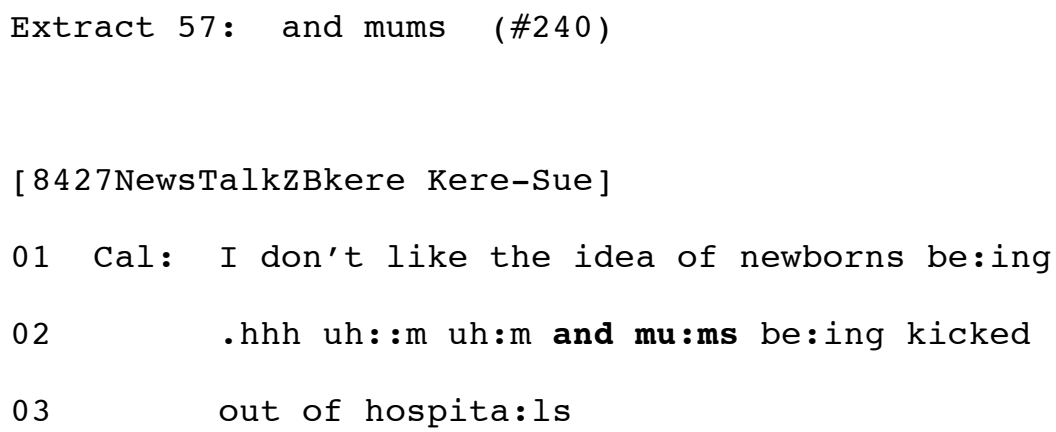

In Extract 58, a speaker is talking about the recipient's family doctor (whom she doesn't know) and refers to this person as she (lines 1 and 2) and subsequently inserts or he between the words she and is in the original version of the TCU.

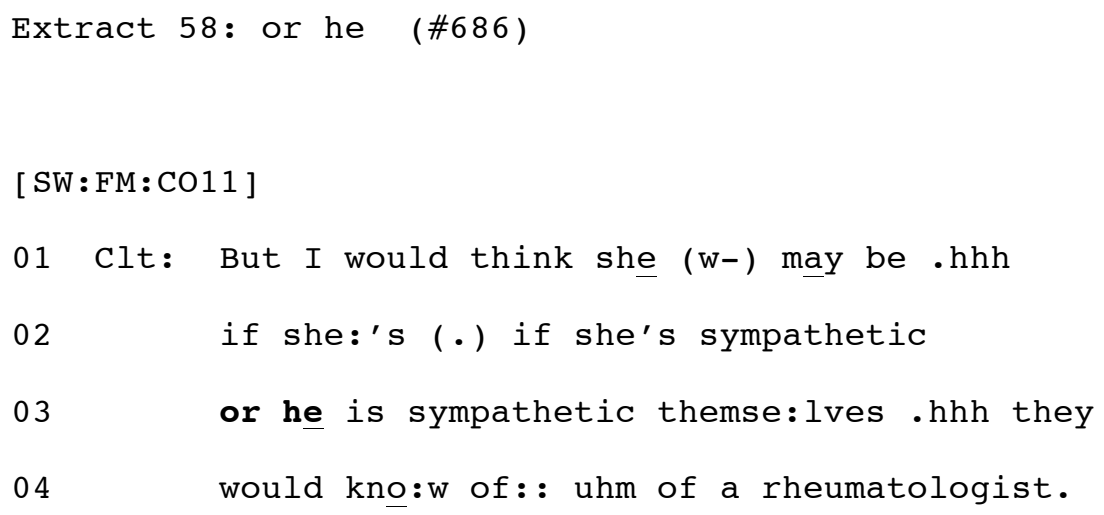

The insertion adds something altogether new - a reference formulation which the conjunction (or) shows to be an alternative (she or he). This repair is attentive to the possibility that she may be incorrect for this referent (but is a co-class member insofar as it is a locally 
subsequent reference form). Whereas 56 and 57 expand the TCU by adding in a new referent (using and), 58 expands the TCU by adding in an alternative way of referring to the same referent (using or).

When TCUs are expanded with additional or alternative reference formulations the inserted material is a multi-word unit: a conjunction (and, or) followed by a noun or pronoun. Other instances include: She's found blood in:: (.) and protei:n in my uri:ne; The public wants: (.) an' overseas visitors to our country want access to .h wh to these lands; he was probably or she $>$ whichever it was $<$ was probably just thinking along those lines.

\section{(b) Expanding with a new formulation of time or place}

In Extract 59, the speaker inserts the time formulation next week (line 3).

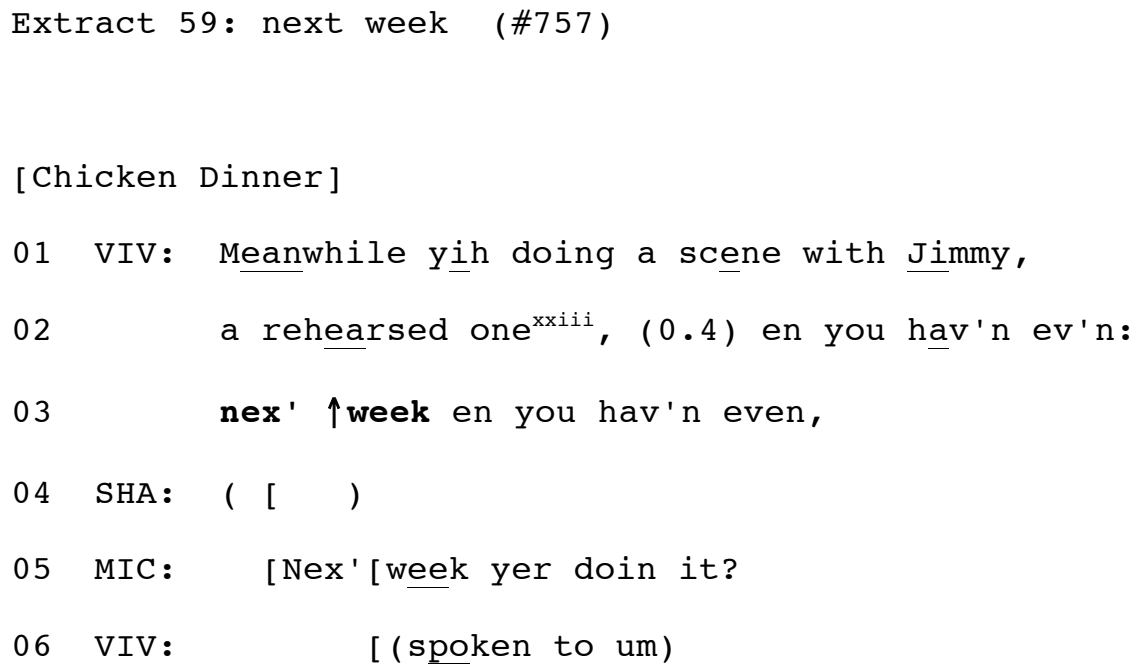

Vivian's insertion of next week renders Shane more culpable by emphasising the imminence of the scene - thereby drawing attention to the length of time during which he has failed to contact Jimmy (from whenever it was that the scene was first proposed until its upcoming production next week). (See Extract 14 for another instance of inserting a time formulation.) 
In Extract 60, a speaker inserts the place formulation in the bedroom:

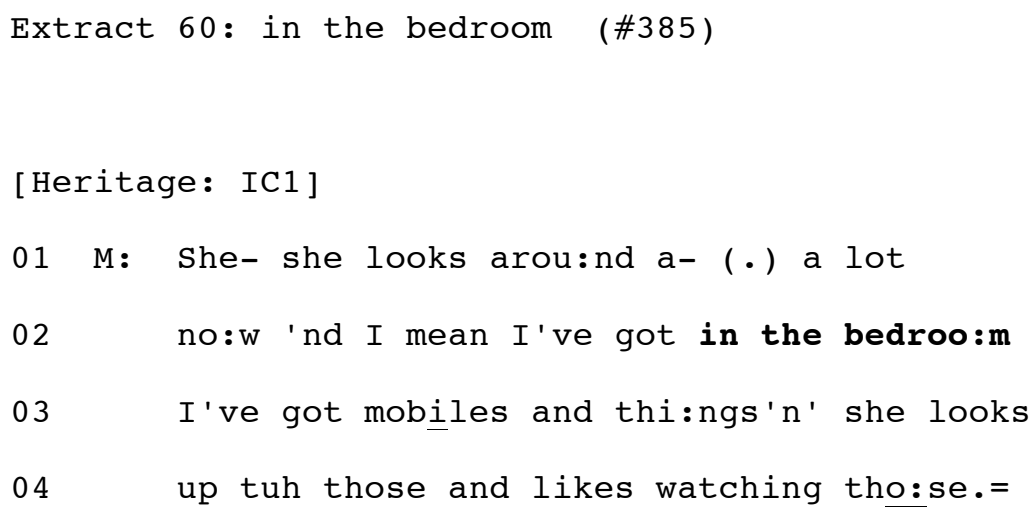

Inserting time or place formulations is commonly done with a multi-word (adverbial) unit often a prepositional phrase. Other inserted formulations of time and place include: that night, at that point, at the moment, later on, in my fifties, in the old days, in the Victorian era, many years ago, for the last nine months, from here.

\section{(c) Expanding with other items}

Other insertions which expand the TCU include: list launchers (e.g. $\underline{A}$ (as in a-b-c), Extract 61, line 3, first, first of all); quotatives (e.g. quote, Extract 62, line 2); courtesy terms (e.g. please, Extract 63, line 7); collective nouns (a par-a set of parents); container nouns (the mubasket of mushrooms); fillers (e.g. you know, sort of), address terms (e.g. Nancy); and interjections (e.g. God!). Space constraints preclude a detailed analysis of these (relatively rare) insertions. 


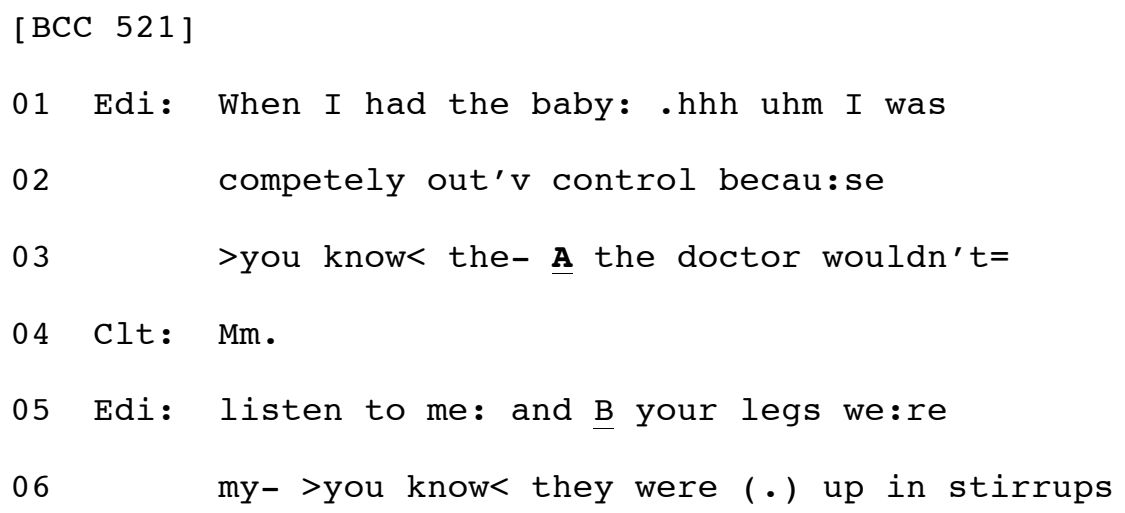

In sum, then, we have shown that, in addition to their key actions of specifying and intensifying, insertion repairs can be used to describe, to correct, and to adjust a formulation, and also to expand an on-going TCU by adding in new material. 


\section{Conclusion}

As we have seen, insertion repair is a practice in which speakers override the preference for progressivity by stopping their talk at a point where it cannot be possibly complete to insert something else into it. We began this investigation with a question from Schegloff (2008): "What sorts of things... warrant such an override, warrant such a marked usage?". By analysing a large number of insertion repairs culled from a wide range of English-language data sets, we have been able to map out both the technology whereby insertion repair is normally accomplished (and some of the diversity of that technology) and also the 'sorts of things' that warrant its use.

The 'sort of thing' that gets inserted is not readily describable primarily with reference either to semantics or to grammar. We have found that single adjectives (and, amongst those, what are sometimes called 'defining adjectives') constitute the most commonly inserted material. However, as far as we can tell, there is nothing at the level of either semantics or grammar that can not be inserted: we have shown instances in which the inserted material is a prefix or initial word component (e.g., 16-20), a multi-word unit (e.g., 13-15), an adverb (e.g., 3,6), noun (e.g., 9) proper name (e.g., 21), initialization (e.g., 22), preposition or prepositional phrase (e.g., 39, 40, 60), or other grammatical form (e.g., 61-63). ${ }^{\text {xiv }}$

The 'sort of thing' that gets inserted is more readily describable at the level of action: that is via an analysis of what it is that the speaker accomplishes by stopping their talk-in-progress to go back and insert just that item at just that point: why that now? We identified the key action that inserting is used to do as specifying (to differentiate or to display relevance). We showed that inserting is also used to do intensifying. Insertion repair is especially suited for these 
tasks because (by definition, and distinctively among repair operations) it modifies an original formulation, and retains it as part of the repair solution, instead of deleting it or replacing it.

When speakers replace a formulation, they take the position that it was 'wrong' - for example, because it indexed the wrong referent (as when Bill is replaced with Bud in And Bill-an'Bud got do:wn); or because it indexed the right referent inappropriately, as when feller is replaced with man in This feller I have- (nn) 'felluh' this ma:n (both examples from Schegloff, Jefferson and Sacks, 1977: $371 \& 376)$. By contrast, insertion repair does not substitute one referent or another, or one formulation for another: instead it retains and builds on the original formulation - mostly inserting material that renders the original formulation more specific or precise (specifying), or which strengthens it (intensifying).

Sometimes a repair is just a repair: that is, it is properly understood as dealing with a possible trouble in speaking, hearing or understanding the talk. In our collection of insertion repairs, those doing specifying to differentiate and those doing correcting are simple repairs of this type. But, as is well established, repair can be used in the service of a range of other actions, beyond simply repairing problems of speaking, hearing and understanding the talk (Jefferson, 1974). Research on other-initiated repair ${ }^{\mathrm{xxv}}$ shows that when speakers initiate repair using an 'open'-class repair initiation (e.g. huh? what?) this can signal misalignment or disaffiliation rather than simply a problem in understanding (Drew, 1997): and there is a systematic basis for this use, namely that in breaking contiguity between initiating and responsive actions, other-initiated repair embodies a canonical feature of dispreference (Schegloff, 2007: 100-6). We have shown that when speakers initiate repair on their own talk in order to insert something new into it (self-initiated insertion repair), this is very often done in the service of either specifying to display the relevance of the referent (as when Islamic is inserted before chap to credential a practitioner of Yunani medicine [26]); or intensifying (as when really is 
inserted before nice to intensify an appreciation [38]). There is a systematic basis for these uses of insertion repair in the service of the interactional-task-at-hand, namely that inserting modifies rather than deletes or replaces the original formulations. The actions of specifying and intensifying are accomplished not only by the material that is inserted (e.g. by the words Islamic and really) but also by the fact that this material is inserted, i.e. that it becomes part of the turn-in-progress as a result of a repair operation. The interactional 'meaning' of a repaired TCU is derived in part from the fact that a speaker has overridden the preference for progressivity to go back and insert something, such that the work for a recipient (as for us as analysts) is to figure out how what is inserted is of sufficient relevance to warrant this override.

Our findings on the use of insertion repair to display the relevance of the referent to the action of the turn also contribute to the literature on practices of referring and membership categorisation devices (Sacks and Schegloff 1979; Schegloff, 1996; 2007a,b). When speakers insert material that identifies a referent as being one of a type or category of referents - as is very commonly the case in specifying insertion repairs - then the relevance of that category (to the speaker) is apparent, simply from the fact that the repair was done. It is however up to the recipient (and to us as analysts) to figure out how the referent's membership in that category is relevant. Sometimes, as we have seen, the category is relevant only because it serves to differentiate one referent from another. So, for example, $N C T$ as a membership category for antenatal teacher [22] may well be inference-rich for these participants (e.g. independent of the hospital, likely to emphasise an informed participative approach etc.) but none of those inferences is analyzably relevant here. The insertion of NCT is simply to achieve adequate reference. On other occasions the category is relevant - and shown to be relevant - because (in the absence of any competing referents) it analyzably contributes to the local action of the turn, and it does so independently of broader cultural inferences that may 
also attach to the particular membership category used. So, for example, Islamic as a membership category for chap [26] is likely - in a post-9/11 world - to be inference-rich in a variety of ways, none of which is analyzably relevant here, where the category is demonstrably employed to contribute to the report of the referent's healing powers.

Finally, despite the close relationship we have identified between the practice of insertion repair and the key actions it is often used to perform (specifying and intensifying), it is clear that inserting can be used to do other actions (we have identified these as describing, correcting, adjusting, and expanding) and it is also apparent that the actions of specifying and intensifying can be accomplished using other repair operations. ${ }^{\text {xxvi }}$ Future research might profitably explore the systematic practices and actions associated with other repair operations (replacing, deleting, reformatting, etc.), thereby enabling a better understanding of the relationship of grammar and action (Ochs, Schegloff and Thompson, 1996) and of how recipients might select from between different repair operations in the service of managing problems of speaking, hearing and understanding; or of contributing to the interactional taskat-hand. 


\section{References}

Ansell, M. (2000) English Grammar. 2nd. ed. Published online at $<$ http://www.fortunecity.com/bally/durrus/153/gramdex.html $>$ (Accessed 31 October 2009)

Curl, T.S. and Drew, P. (2008) Contingency and action: A comparison of two forms of requesting. Research on Language and Social Interaction, 41(2): 129-153.

Drew, P. (1997) 'Open' class repair initiators in response to sequential troubles in conversation. Journal of Pragmatics, 28: 69-101.

Drew, P. (2003) Precision and exaggeration in interaction. American Sociological Review 68(6): 917-938.

Drew, P. (2006) Misalignments in "after-hours" calls to a British GP's practice: A study in telephone medicine. In J. Heritage and D.W. Maynard (Eds) Communication in Medical Care. Cambridge: Cambridge University Press. pp. 416-444.

Edwards, D. (2000) Extreme case formulations: Softeners, investment and doing nonliteral. Research on Language and Social Interaction 33(4): 347-373.

Enfield, N.J. and Stivers, T. (Eds) (2007) Person Reference in Interaction: Linguistic, Cultural and Social Perspectives. Cambridge: Cambridge University Press.

Heritage, J. (2002) The limits of questioning: Negative interrogatives and hostile question content. Journal of Pragmatics, 34: 1427-46.

Heritage, J. and Raymond, G. (2005) The terms of agreement: Indexing epistemic authority and subordination in talk-in-interaction. Social Psychology Quarterly, 68(1): 15-38.

Hommerberg, C. and Tottie, G. (2007) Try to or try and?: Verb complementation in British and American English. ICAME Journal, No.31: 45-64. 
Jefferson, G. (1974) Error correction as an interactional resource. Language in Society, 2: 181-99.

Jefferson, G. (1996) On the poetics of ordinary talk. Text and Performance Quarterly, 16(1): $1-61$.

Labov, W. and Fanshel, D. (1977) Therapeutic Discourse: Psychotherapy as Conversation. New York: Academic Press.

Kärkkäinen, E. (2003) Epistemic stance in English conversations: A description of its interactional functions, with a focus on I think. Amsterdam: Benjamins.

Lerner, G.H. and Kitzinger, C. (2007a) Introduction: Person-reference in conversation analytic research. Discourse Studies, 9: 427-433. (Special Issue)

Lerner, G.H. and Kitzinger, C. (2007b) Extraction and aggregation in the repair of individual and collective self-reference. Discourse Studies, 9: 526-558.

Lerner, G.H. and Kitzinger, C. (2010) Repair prefacing in the organization of same-turn selfrepair. Paper to be presented at the International Conference on Conversation Analysis. Mannheim, July.

Lerner, G.H., Kitzinger, C. and Raymond, G. (2009) Some sources of cascading troubles in the organization of repair. Paper to be presented at the 95th Annual Convention of the National Communication Association, Chicago, November.

Ochs, E., Schegloff, E.A. and Thompson, S.A. (1996) Interaction and Grammar. Cambridge: Cambridge University Press.

Pomerantz, A. (1984) Agreeing and disagreeing with assessments: Somme features of preferred/dispreferred turn shapes. In J.M. Atkinson and J. Heritage (Eds), Structures of Social Action: Studies in Conversation Anakysis. Cambridge: Cambridge University Press. pp. 57-101. 
Pomerantz, A. (1986) Extreme case formulations: A way of legitimizing claims. Human Studies 9: 219-229.

Sacks, H. (1972) An initial investigation of the usability of conversational data for doing Sociology. In D.N. Sudnow (Ed.), Studies in Social Interaction. New York: The Free Press. pp. 31-74. (The Search for Help)

Sacks, H. and Schegloff, E.A. (1979) Two practices in the organization of reference to persons in conversation and their interaction. In G. Psathas (Ed.(, Everyday Language: Studies in Ethnomethodology. New York: Irvington. pp.15-21.

Schegloff, E.A. (1972) Notes on a conversational practice: Formulating place. In D.N. Sudnow (Ed.), Studies in Social Interaction. New York: The Free Press. pp.75-119. Schegloff, E.A. (1979) The relevance of repair to syntax-for-conversation. In T. Givon (Ed.), Discourse and Syntax. New York: Academic Press. pp. 261-286.

Schegloff, E.A. (1987) Analyzing single episodes of conversation: An exercise in conversation analysis. Social Psychology Quarterly, 50(2): 101-114.

Schegloff, E.A. (1996) Some practices for referring to persons in talk-in-interaction: A partial sketch of a systematics. In: B. Fox (Ed.), Studies in Anaphora. Amsterdam: John Benjamins. pp. 437-485.

Schegloff, E.A. (2000a) When 'others' initiate repair. Applied Linguistics, 21(2): 205-243.

Schegloff, E.A. (2000b) On granularity. Annual Review of Sociology, 26:715-20.

Schegloff, E.A. (2003) The surfacing of the suppressed. In P. Glenn, C.D. LeBaron and J. mandelbaum (Eds), Studies in Language and Social Interaction. Mahwah, N.J.: Lawrence Erlbaum. pp. 241-262.

Schegloff, E.A. (2007a) Categories in action: Person-reference and membership categorization. Discourse Studies, 9: 433-462. 
Schegloff, E.A. (2007b) Sequence Organization in Interaction. Cambridge: Cambridge University Press.

Schegloff, E.A. (2007c) A tutorial on membership categorization. Journal of Pragmatics, 39: $462-482$.

Schegloff, E.A. (2008) Ten operations in self-initiated, same-turn repair. Paper presented at workshop on "Repair and intersubjectivity in talk and social interaction", University of Toronto, March.

Schegloff, E.A. (2009) On the boundaries of a turn's space: Evidence from repair and increments. Paper to be presented at the 95th Annual Convention of the National Communication Association, Chicago, November.

Schegloff, E.A., Jefferson, G. and Sacks, H. (1977) The preference for self-correction in the organization of repair in conversation. Language, 53: 361-382.

Schegloff, E.A. and Lerner, G.H. (2009) Beginning to respond: Well-prefaced responses to Wh-questions. Research on Language \& Social Interaction, 42(2): 91-115.

The Times (2009) Lesbian soldier has payout cut. 15 October: 22.

Author 1 (2010, in press) Gender, routinization and recipient design. In S. Speer and E. Stokoe (Eds), Conversation and Gender. Cambridge: Cambridge University Press. Author 1 and Kitzinger, C. (2006) Surprise as an interactional achievement: Reaction tokens in conversation. Social Psychology Quarterly, 69(2): 150-182. 
(1) Hyla and Nancy

(2) BBC Radio 4, PM, 4.6.09

(3) Holt SO88 (II) 1.3

(4) BCC204 Vita

(5) EGC Connie 8Q

(6) DEC-1-2-08 (MUMPS)

(7) BCC004 Donna

(8) $\mathrm{BCC}$

(9) WCSNZE

(10) BBC Radio 4, Open Book

(11) BBC Radio 4, PM, 26.11.08

(12) PP103 Kate

(13) BCC02 Brenda

(14) PP12 Heather

(15) HB67 Janet

(16) BCC259

(17) BCC552

(18) SW:FM:C011

(19) Holt 1.1

(20) TCIB.2.3 


\section{Notes}

${ }^{\mathrm{i}}$ Sources of the short data extracts in this Introduction and in the following section on 'Technology' are given in Appendix 1.

${ }^{\text {ii }}$ For pragmatic reasons to do with the way the collection was compiled, there are some gaps in the consecutive numbering of instances in our collection - so data tags may identify individual instances using numbers well in excess of 500 .

iii These include many of the classic CA data sets (e.g. TG, Hyla \& Nancy, NB, SBL, Virginia, Chicken Dinner) as well as more contemporary data sets (e.g. the Wellington Corpus of Spoken New Zealand English; the Holt corpus of British-English phone calls). They also include data drawn from calls to a number of telephone helplines (e.g. Birth Crisis, Home Birth, Pelvic Partnership, The Fibromyalgia Association, and the NZ Electricity and Gas Commissioner's Office); and from interview, documentary and talk-back programmes on British, New Zealand and US radio. The practices and actions documented in this article apply across these data sets. (We have not undertaken systematic comparisons, but have not observed any differences in the use of insertion between, for example, British, NZ and US data; or between classic and contemporary data; or between institutional and ordinary conversational data.)

iv Although the technology of same-turn repair and transition space repair obviously differs, the actions done by insertion repair in these different positions are - as far as we know - the same. Although most of the data extracts displayed here are instances of same-TCU repair, our collection also includes instances of 'specifying', 'intensifying' (and other actions described below) in which insertion repair is initiated in the transition space. 
${ }^{\mathrm{v}}$ Note that in (8), what the recipient hears in real time is this chap this and it is this discontinuity which provides the initial signal that repair may be underway, and not the subsequent sound stretch on the repeated this (which may be heard as initiating a second repair: a word search for the appropriate characterization of the chap).

vi They can repeat more - sometimes a great deal more. The pre-frame in the case analyzed by Schegloff (1987) (he's about the only regular <he's about the only good regular out there) is exceptional; we have no pre-frames this extensive in our collection, but see Extract 17 for a two-word repeat constituting a pre-frame.

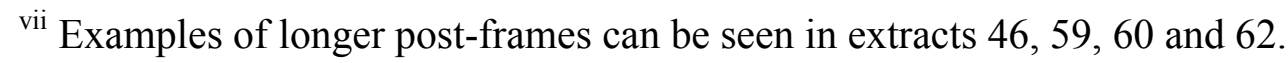

viii Other multi-word insertions in our collection include: one-to-one (care), five-year-old (girl), stamped addressed (envelope), man-made (changes), parole board (rules), under these circumstances, in the Victorian era.

${ }^{\text {ix }}$ As with insertion repair more generally, items inserted to modify a reference formulation and thereby specify the referent range across a variety of grammatical forms, including adjectives (most commonly), but also nouns and compounds. So-called 'defining adjectives' (Ansell, 2000) are particularly well-suited to the work of specification.

${ }^{\mathrm{x}}$ Particular thanks to Manny Schegloff for helping us more clearly to articulate the key feature of our findings here.

${ }^{x i}$ Cary is an abbreviation of the name of a village, in Somerset, England (Castle Cary). North Cadbury is another village in the same county (the county in which both participants live). xii The National Childbirth Trust is the UK's leading charity to parents "supporting you to become the parent you want to be" and - according to its website $<$ www.nctpregnancyandbabycare.com $>$ - providing "support and evidence-based information in pregnancy, birth, and early days of parenthood". Its antenatal classes are a popular alternative (or addition) to National Health Service (i.e. government-run) antenatal classes. It 
appears that Jennifer had attended classes of both types, and that her midwife had run the latter.

xiii Here's what she says:

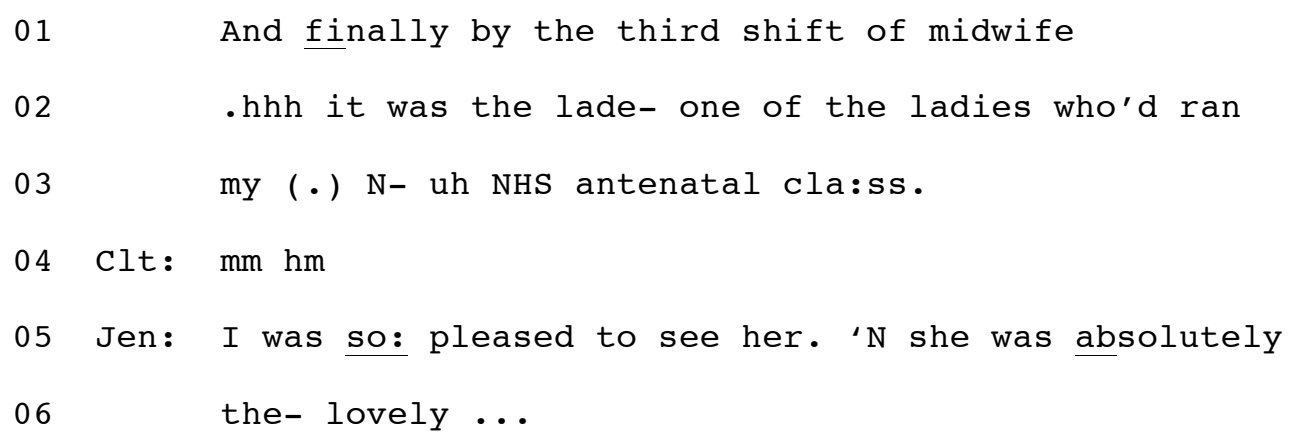

${ }^{\text {xiv }}$ Here is the earlier reference:

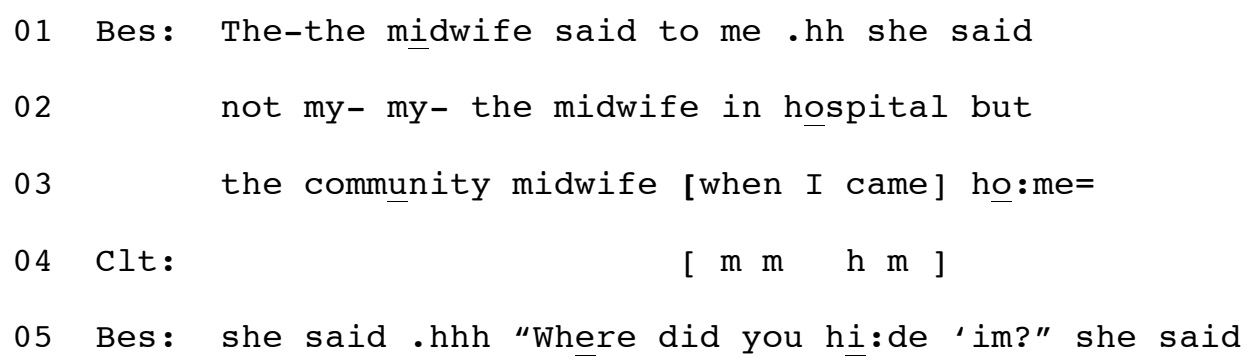

Another insertion repair earlier in the same conversation makes the same differentiation between the two midwives:

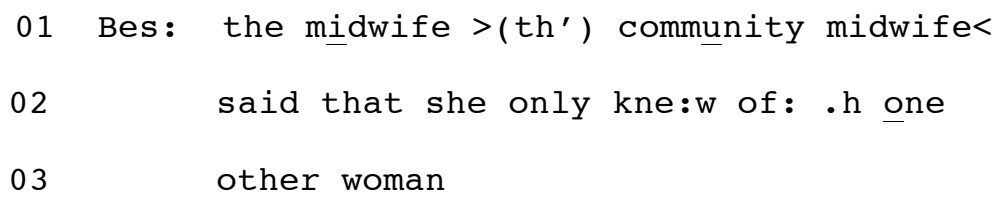

${ }^{\mathrm{xv}}$ We have another instance in our collection of specifying analyzably done in the service of credentialling a practitioner. In this case, a woman expecting triplets is forestalling dietary advice from a helpline counsellor. She reports that she has already received such advice from a consultant, subsequently specified as a multiple-birth consultant, thereby credentialing him as well-qualified to advise her.

xvi 'Gorilla Kingdom', opened in Spring 2007, is the largest investment London Zoo has made in 40 years. According to a zoo spokesperson, quoted in The Observer on 18 March 2007, the GBP 5 million enclosure is "designed to mimic the features gorillas would have in a natural 
habitat" and to "give them the enrichment they need". Such enclosures are a rarity in British zoos.

xvii Other examples of specification in our collection include: the insertion of blind before date (1), first before husband, cotton before wool, calamine before lotion, stool before specimen, menstrual before cycle, hard before copy, Sony before cassette recorder, ex- before consultant (16) and before wife, re- before pay and before discovered. After insertion, laws are specified as drug laws (10), and as Jewish laws; the office as the publisher's office and as the accounts office; tax as income tax and as Council tax; reading as background reading and as meter reading.

${ }^{\text {xviii }}$ Extreme case formulations have been described as 'factually brittle' (Edwards, 2000: 352), with the potential to meet with 'recipients' skepticism' (Drew, 2003: 933) compared with more qualified versions. Whereas Edwards and Drew explore how speakers sometimes repair or back down from extreme case formulations, in our data, repair is used to construct them. ${ }^{\text {xix }}$ For an analysis of insertings of just, see Schegloff (2008).

${ }^{x x}$ Also analyzed by Schegloff (2007a).

xxi We have already seen that pre-frames can be amended to fit the new grammatical structure of a repaired turn (Extract 2, where $a$ is amended to $a n$ ). When auxiliary or catenative verbs are inserted the post-frame is systematically amended to fit the new grammatical structure that is the upshot of the insertion. This often means using the infinitive: so getting becomes to get (51), explain becomes to explain (53), waited becomes to wait (55) and so on. Although at first glance, it might appear that the word to (or and in 52 - try and is a common colloquial version of try to in British English, [Hommerberg and Tottie, 2007]) is part of what is inserted (since to - or and - was not in the original version but is there in the repaired version) the word to (or and) is properly considered not as part of the insertion itself but as part of the amended post-frame. 
xxii Thanks to Alice Harvey for permission to use this data.

xxiii This is one of two repairs; the other is her parenthetical a rehearsed one (line 2). The defining adjective rehearsed specifies the scene (as rehearsed rather - presumably - than ad lib) but the specification is done differently from those described earlier via a parenthetical and as an insertion repair.

${ }^{\text {xxiv }}$ Although specifying is very often accomplished with defining adjectives (e.g. blind in blind date [1]; Cary in Cary cemetery [21]) it can also be done with modifiers (e.g. other in other shoulder [25]) and prefixes (e.g. micro- and neuro- in microhabitats [29] and neurochemicals [18]); and although intensifying is very often accomplished with adverbs, it can also be done with idioms (e.g. on earth [43], in the world [44]). In the domain of insertion repair, then, there is no one-to-one relationship between grammar and action. ${ }^{x x v}$ A range of different actions that self-initiated repair can be used to accomplish have been identified on a case-by-case basis (e.g. mitigating a display of entitlement [Curl and Drew, 2008]; avoiding an excessively conducive form of question design [Heritage, 2002]; incorporating "a reference to an otherwise disattended utterance by another participant" [Schegloff, 1987: 111]). These analyses have not, however, paid attention to the relationship between the actions repair is used to do, and the type of repair operation (inserting, deleting, replacing, reformatting etc.).

${ }^{x x v i}$ For example, specification can be accomplished using the repair operation Schegloff (2008) calls parenthecizing, as when the speaker in the extract shown in footnote 11 differentiates between her community midwife and her midwife in hospital with a 'not $\mathrm{X}, \mathrm{Y}$ ' parenthetical repair. And intensifying can be done with transition-space replacement repair as in this extract, where the speaker replaces quite with very to modify severe:

[PP 666 ]

01 Clt: you've ha:d hh (0.6) (th) quite seve:re S-P-D 
